\title{
Cross-slip of long dislocations in FCC solid solutions
}

\author{
Wolfram Georg Nöhring ${ }^{\mathrm{a}, 1, *}$, W. A. Curtin ${ }^{\mathrm{a}}$ \\ ${ }^{a}$ Institute of Mechanical Engineering, École Polytechnique Fédérale de Lausanne, EPFL STI IGM Station 9, CH-1015 Lausanne
}

\begin{abstract}
Cross-slip of screw dislocations is a dislocation process involved in dislocation structuring, work hardening, and fatigue. Cross-slip nucleation in FCC solid solution alloys has recently been shown to be strongly influenced by local fluctuations in spatial arrangement of solutes, leading to a statistical distribution of cross-slip nucleation barriers. For cross-slip to be effective macroscopically, however, small cross-slip nuclei $(40 \mathrm{~b})$ must expand across the entire length of typical dislocation segments $\left(10^{2}-10^{3} \mathrm{~b}\right)$. Here, a model is developed to compute the relevant activation energy distribution for cross-slip in a random FCC alloy over arbitrary lengths and under non-zero Escaig and Schmid stresses. The model considers cross-slip as a random walk of successive flips of adjacent $1 b$ segments, with each flip having an energy consisting of a deterministic contribution due to constriction formation and stress effects, plus a stochastic contribution. The corresponding distribution is computed analytically from solute-dislocation and solute-solute binding energies. At zero stress, the probability of high activation energies increases with dislocation length. However, at stresses of just a few MPa, these barriers are eliminated and lower barriers are dominant. For increasing segment length, the effective energy barrier decreases according to a weak-link scaling relationship and good analytic predictions can be made using only known material properties. Overall, these results show that the effective cross-slip barrier in a random alloy is significantly lower than estimates based on average elastic and stacking fault properties of the alloy.
\end{abstract}

Keywords: screw dislocation, cross-slip, face-centered cubic crystals, solid solution, stochastic model

\section{Introduction}

In many engineering alloys, some fraction of the alloying elements remain in solution as substitutional solutes. The average effect of solutes leads to changes in the bulk material properties, especially the lattice constant, elastic constants, and stacking fault energies. Solutes also interact with dislocations to inhibit or modify dislocation motion. For non-diffusive solutes, solute/dislocation interactions lead to solute strengthening. For solutes that diffuse readily, solute/dislocation interactions can lead to solute drag and the associated creation of Cottrel atmospheres, dynamic strain aging, and Suzuki segregation to stacking faults. Understanding the role of solutes, and especially the interactions between solutes and dislocations is useful for development of new engineering alloys with specific performance requirements.

One of the fundamental processes of dislocation motion is cross-slip of screw dislocations, i.e. the change of dislocation slip plane. Cross-slip is a versatile mechanism. It enables the dislocation to bypass obstacles, to form new sources on adjacent planes, or to annihilate other dislocations. In FCC metals, in particular, cross-slip plays a role in, for example, dislocation structuring [1, 2], and work hardening and recovery [3-5]. Mobile screw dislocations in FCC are dissociated into pairs of Shockley partial dislocations with mixed character, so that the

\footnotetext{
${ }^{*}$ Corresponding author

Email address: wolfram.noehringeimtek.uni-freiburg.de (Wolfram Georg Nöhring )

${ }^{1}$ Now at: Department of Microsystems Engineering, University of Freiburg, Georges-Köhler-Allee 103, DE-79110 Freiburg
}

partial dislocations are not able to cross-slip. Cross-slip mechanisms for FCC crystals are thus more complex than in BCC crystals where dislocations do not dissociate. Many previous studies focused on the mechanism and energy of cross-slip in pure metals, see for instance the review [ 6] and Refs. [7-10]. The most prominent mechanism is the Friedel-Escaig [11, 12] mechanism. The first step of this mechanism is the formation of a point constriction of the two partials. The dislocation then re-dissociates onto the cross-slip plane, and expands to create a lenticular segment on that plane. Fig. 1a) shows the typical transition state as found using an atomistic transition path calculation [13]; note that the segment on the cross-slip plane (blue) is bounded by two constrictions. The segment then grows by lateral movement of the constrictions.

Cross-slip is a thermally activated mechanism and it has been shown $[9,14]$ that the rate of cross-slip can be described by an Arrhenius expression,

$$
r(T)=\operatorname{vexp}-\frac{\Delta E_{\mathrm{act}}}{k_{B} T},
$$

where $T$ is temperature, $k_{B}$ is Boltzmann's constant, $v$ is a prefactor and $\Delta E_{\text {act }}$ is the activation energy. Analytic models [12] of this process in elemental FCC metals show that the energy barrier for cross-slip scales as $\mu^{2} b^{4} / \gamma_{\mathrm{f}} \log \left(\overline{3} \mu b / 8 \pi \gamma_{\mathrm{sf}}\right)$ where $\mu$ is the shear modulus, $\chi_{\mathrm{f}}$ is the stacking fault energy, and $b$ is the magnitude of the Burgers vector of a $a / 2 h 110 i$ dislocation, i.e. $a / \overline{2}, a$ being the lattice parameter. For materials like $\mathrm{Cu}$ and $\mathrm{Ni}$, with low $\chi_{\mathrm{f}}$, the activation barriers from atomistic calculations and analytical models are very large, $>1.5 \mathrm{eV}$, making 
cross-slip very slow at room temperature. Since experimental observations of cross-slip indicate that it is more prevalent than such barriers suggest, there have been recent analyses of "heterogeneous" cross-slip mechanisms initiating at, for example, jogs $[15,16]$ or forest dislocations [17-19].

In contrast, there are few studies of solute effects on crossslip [20-24]. Thus, we recently performed a comprehensive atomistic study of cross-slip in $\mathrm{Al}-\mathrm{Mg}, \mathrm{Cu}-\mathrm{Ni}$ and $\mathrm{Ni}-\mathrm{Al}$ solid solutions [13]. Using atomistic transition path calculations, we demonstrated that cross-slip nucleation, which occurs over scales of $\approx 40 b$, is strongly influenced by the fluctuations in the spatial distribution of solutes over the scale of the critical nucleus. While the average barrier is comparable to that computed using the average modulus and stacking fault energy, there is a broad distribution of activation energies around the average. Since cross-slip is thermally-activated, nucleation can thus occur in regions where the local activation energy is significantly below the average value. The nucleation of screw dislocations on the cross-slip plane is thus much faster than expected based on average material properties. However, it is not sufficient to consider only nucleation of cross-slip. In order to meaningfully impact plastic deformation, the nucleus either has to expand laterally, so that eventually the whole dislocation cross-slips, or the initial cross-slip event must enable some other transformation of the dislocation, e.g. formation of a source by bow-out on an adjacent plane. In the former case, the cross-slipped length increases from the nucleation length of ca. $40 \mathrm{~b}$ to the typical length of dislocations in the network, which is on the order of $1000 \mathrm{~b}$ for a deformed metal with a dislocation density ${ }^{2}$ of $10^{13} \mathrm{~m}^{-2}$. In the latter case, the cross-slipped length is shorter, but must still be on the order of $\mu b / \tau$, where $\tau$ is the applied stress, so that the bow-out in the adjacent plane is stable. In Ref. [13], we speculated that lateral expansion could occur without difficulty, but this is incorrect - the expanding cross-slipped dislocation could encounter energetically unfavorable environments that require further thermal activation. Therefore, the fundamental work of Ref. [13] must be extended to encompass the full process from nucleation at the scale of $\approx 40 b$ to full cross-slip over scales $>1000 b$. The purpose of this paper is to make such an extension and draw meaningful conclusions about the true role of solutes in controlling cross-slip in random FCC alloys. This is achieved using a random-walk-like model. We view the crossslip process as a discrete sequence of flips of successive unit dislocation segments from the glide to the cross-slip plane. The energy change upon cross-slip of each segment has a random and a deterministic part. The former reflects the random change in solute binding energies, and the latter the energy needed for constriction formation, as well as stress effects. We provide expressions for these energy changes, which then allows us to calculate distributions of energy barriers for cross-slip of long dislocations.

The rest of the paper is structured as follows. In Sec. 2,

\footnotetext{
${ }^{2}$ A standard estimate for the average dislocation spacing $L$ at dislocation density $\rho$ is $L=1 /{ }^{\bar{\rho}}[25$, p. 20]. For a cellular or mesh-like dislocation microstructure, $L$ is also an estimate for the typical segment length. For example, in Ni $b=2.48 \AA$, hence $L / b=1270$ at $\rho=10^{13} \mathrm{~m}^{-2}$.
}

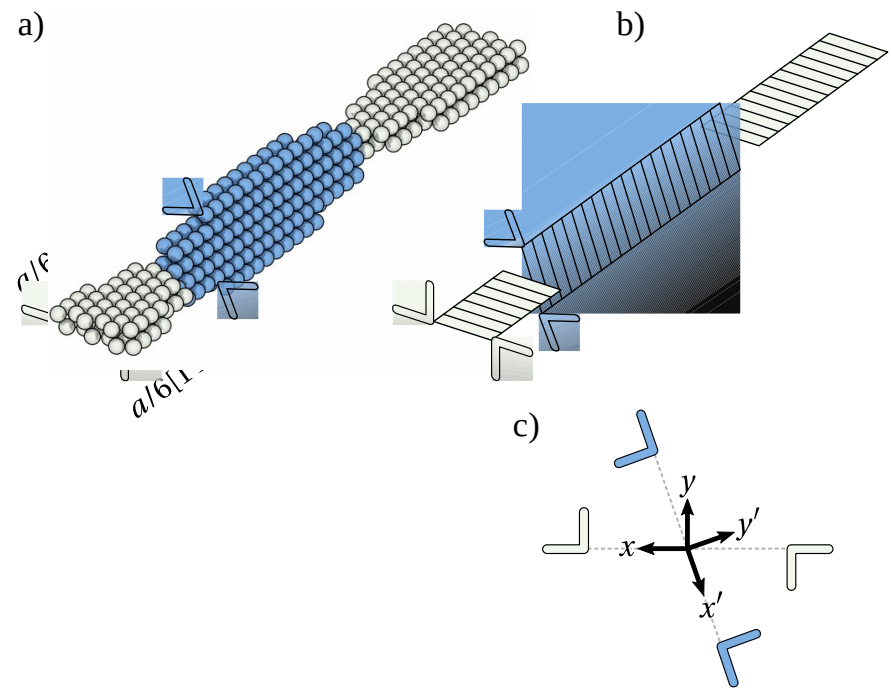

Figure 1: a) Cross-slip transition state in a Ni-15at.\% $\mathrm{Al}$ average alloy, from an atomistic transition path calculations reported in Ref. [ 13]; only atoms that don't have regular FCC coordination are shown; gray: segment on the glide plane; blue: cross-slip plane; atoms visualized using O VITO [26]. b) Simplified picture of the process, as used in the random walk model; the dislocation is partitioned into $N_{b}$ segments of $1 b$ length; cross-slip is the discrete process of moving the segments one by one from the glide to the cross-slip plane.

we briefly summarize Ref. [13] and the important underlying analysis of changes in solute/dislocation binding energies during cross-slip, extending the results beyond those presented in Ref. [13]. In Sec. 3 we then introduce the random walk model for studying the nucleation and lateral expansion of a nucleated cross-slip segment, for zero stress and for non-zero Escaig and Schmid stresses. In Sec. 4, we validate the random walk model predictions of the activation barrier by comparisons with fully atomistic results at the short nucleation length of $\approx 40 \mathrm{~b}$ for zero stress and non-zero stresses. In Sec. 5, we then use the validated random walk model to study long dislocations, which cannot be treated using atomistic calculations, again for zero stress and non-zero stresses. Finally, in Sec. 6, we summarize the broad general findings and discuss their implications for cross-slip processes in metal alloys.

\section{Cross-slip barriers in random alloys; correlation with so- lute/dislocation interactions}

In numerical studies of cross-slip in both elemental FCC metals and homogeneous "average alloy" representations that eliminate all stochastic features [27, 28], cross-slip is observed to occur by the Friedel-Escaig model with an activation energy barrier $\Delta E_{\text {act }}$ controlled by the elastic modulus and stacking fault energy. However, in explicit random alloys, the variations in local spatial arrangement of solutes create local variations in the cross-slip barriers, leading to a statistical distribution of activation barriers around the average value found in the homogeneous representation. Since cross-slip is thermally activated, those barriers below the average value enable the nucleation of cross-slip at rates far exceeding the average rate. It is thus crucial to understand both the statistical distribution of activation barriers 
and whether cross-slip segments at the typical nucleation size $(\approx 40 \mathrm{~b})$ can expand laterally to create fully cross-slipped long segments.

In our previous work [13], we addressed the statistical distribution of $\Delta E_{\text {act }}$ at the length scale of $40 \mathrm{~b}$. We found that there is a correlation between the values of $\Delta E_{\text {act }}$ and the energy difference $\Delta E_{\text {end }}$ between the initial and final states, i.e. the dislocation segment fully dissociated on the glide or cross-slip plane, respectively. The correlation was

$$
\Delta E_{\mathrm{act}} \approx \Delta E_{\mathrm{act}, \mathrm{avg}}+0.5 \Delta E_{\mathrm{end}} .
$$

A typical example of the correlation is shown in Fig. 2, for the $\mathrm{Ni}-15 \% \mathrm{Al}$ alloy. Therefore, an understanding of the statistical distribution of $\Delta E_{\text {end }}$ can provide direct information on the statistical distribution of $\Delta E_{\text {act }}$. We note now that the random walk model developed in this paper actually reproduces the above correlation with no assumptions; Eq. 2 is an outcome of the physical model for evolution of the cross-slip nucleation, as seen also in Fig. 2. Basic considerations, and the random walk model, show that the correlation only holds for the nucleated segment ( $\approx 40 b$ ), and not for much longer segments. Thus, the previous work uncovered a key feature of cross-slip in random alloys but did not present a full picture. However, both the correlation and the random walk model rely on understanding the statistical distribution of energies of $\Delta E_{\text {end }}$. The correlation requires the distribution of $\Delta E_{\text {end }}$ at the scale of $40 b$ while the random walk model requires only the distribution of energies per unit $b$, which is the fundamental unit of the random walk model as described in Sec. 3. Therefore, an accurate prediction of the distribution of $\Delta E_{\text {end }}$ is needed.

In Ref. [13], the distribution of $\Delta E_{\text {end }}$ was predicted as a function of the underlying solute-dislocation and solute-solute binding energies in binary alloys. Good but not excellent agreement with simulations was obtained by including only nearneighbor solute pairs. We thus start this paper by extending the earlier model to include solute pairs out to eight neighbors, and demonstrate excellent agreement with simulation results. The analysis is further generalized to multiple solutes (beyond binary alloys) for future applications to more complex alloys including High Entropy Alloys [29].

In the absence of any Escaig stresses, the average energies of the initial and final dislocation are equal since both are screw dislocations differing only in the plane of dissociation. Thus, in the random alloy, the energy difference $\Delta E_{\text {end }}$ between the initial and final states is due only to the differences in solutedislocation and solute-solute binding energies for the specific spatial arrangement of solutes around the cross-slipping segment. For a given arrangement of solutes, cross-slip causes changes of the relative position of the solute with respect to the dislocation. During cross-slip, the stacking fault ribbon is destroyed on the glide plane and then re-formed on the cross-slip plane. In both steps, the relative position of some pairs of atoms changes. For example, a first-nearest neighbor pair of solutes may become separated or formed in the process. However, only solute pairs where one solute lies above the fault and the other below are affected.

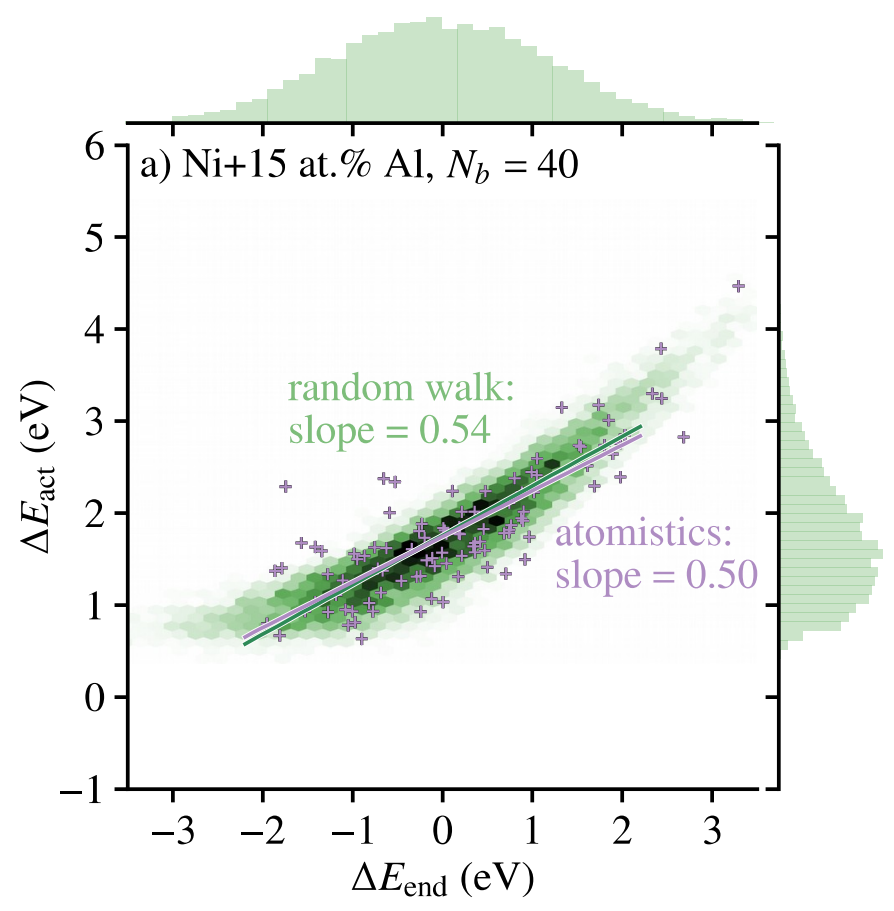

Figure 2: Joint plot of the distribution of activation energy $\Delta E_{\text {act }}$ and end state energy difference $\Delta E_{\text {end }}$ for $\mathrm{Ni}+15$ at. $\% \mathrm{Al}$ and $N_{b}=40$; hexagonal bins: random walk data, color indicates frequency, see marginal distributions; "+": atomistic data; lines: linear regression

The change in solute-dislocation binding energies during cross-slip follows the analysis of solute strengthening in random alloys given by Varvenne et al. B0] following the original work by Leyson and Curtin [31,32]. Consider a slice of material perpendicular to the dislocation line. Define a Cartesian coordinate system with origin at the center of the dislocation, direction $x$ in the glide direction, $y$ normal to the glide plane, and $z$ along the dislocation line, see Fig. 1c). Define a similar coordinate system for the cross-slipped dislocation. We label atomic sites with indices $(i, j, k)$, and define the occupation variable $s_{i j k, X}=1$ if a solute of type $X$ exists at site $(i, j, k)$ and $s_{i j k, X}=0$ otherwise. The spatial positions of solute $(i, j, k)$ in the initial and final configuration are $\left(x_{i}, y_{j}, z_{k}\right)$ and $\left(x_{i}^{0}, y_{j}^{0}, z_{k}^{0}\right)$, respectively. The solute-dislocation binding energy is then denoted as $U_{\mathrm{s}-\mathrm{d}, X}\left(x_{i}, y_{j}, z_{k}\right)$; this is the energy change associated with moving a solute from infinity to the position $\left(x_{i}, y_{j}, z_{k}\right)$. The total energy change of the system due to cross-slip is the sum over all the individual changes in solute-dislocation binding energies of each solute,

$$
\Delta E_{\mathrm{end}, \mathrm{s}-\mathrm{d}, X}=\sum_{i j k} s_{i j k} U_{\mathrm{s}-\mathrm{d}, X}\left(x_{i}^{0}, y_{j}^{0}, z_{k}^{0}\right)-U_{\mathrm{s}-\mathrm{d}, X}\left(x_{i}, y_{j}, z_{k}\right),
$$

For a random alloy, the $s_{i j k}$ are Bernoulli random variables. The average change in energy is thus the average of Eq. 3, and the average is taken only over the $s_{i j k, X}$, yielding $s_{i j k, X}=c_{X}$ where $c_{X}$ is the overall concentration of solute $X$. Recall that the initial and final state of cross-slip are symmetrically equivalent in a pure metal or an average alloy. Hence, for each atomic site $\left(x_{i}, y_{j}, z_{k}\right)$ there is a symmetry-equivalent site $\left(x_{p}, y_{q}, z_{r}\right)$ with opposite contribution in Eq. 3 . Therefore, $\sum_{i j k}\left[U_{\mathrm{s}-\mathrm{d}, X}\left(x_{i}^{0}, y_{j}^{0}, z_{k}^{0}\right)-\right.$ 
$\left.U_{\mathrm{s}-\mathrm{d}, X}\left(x_{i}, y_{j}, z_{k}\right)\right]=0$, and the average energy change is zero. Effects due to discrete solutes thus arise only due to local statistical fluctuations in the actual solute distribution in the alloy. The quantity of interest is thus the standard deviation of the energy change. The solute energies are independent of coordinate $z$ and so for line length $\zeta$ there are $\zeta / b$ identical sites and the statistical quantity $h\left(\sum_{i j k} s_{i j k, X}{ }^{2}\right.$ is $h\left(\sum_{i j k} s_{i j k, X}{ }^{2}=c_{X}\left(1-c_{X}\right) \frac{\zeta}{b}\right.$. The standard deviation of the energy change $\Delta E_{\text {end,s-d, } X}$ is then

$$
\begin{aligned}
& \sigma\left[\Delta E_{\text {end, }, \mathrm{d}, X}\right]= \\
& c_{X}\left(1-c_{X}\right) \frac{\zeta}{b} \sum_{i j} U_{\mathrm{s}-\mathrm{d}, X}\left(x_{i}^{0}, y_{j}^{0}, x_{k}^{0}\right)-U_{\mathrm{s}-\mathrm{d}, X}\left(x_{i}, y_{j}, z_{k}\right)^{2},
\end{aligned}
$$

and the standard deviation of the total solute-dislocation binding energy change is

$$
\sigma\left[\Delta E_{\text {end,s-d }}\right]=S \overline{\sum_{X}^{N_{T}}\left(\sigma\left[\Delta E_{\text {end,s-d, } X}\right]\right)^{2} .}
$$

The change in solute-solute binding energies due to the crossslip process is also zero on average, so that it is again the standard deviation in the energy change that sets the scale of energy changes during cross-slip. A model of the standard deviation is derived in Appendix A. We take into account cutting/formation of the first eight nearest neighbor pairs of the perfect FCC lattice. In a faulted lattice, one can also identify pairs whose distance is not found in the perfect FCC lattice, but these are ignored. Let $U_{\mathrm{s}-\mathrm{s}, X Y, n}$ be the binding energy of anth order pair of solutes $X$ and $Y$. For convenience, write the pair energies of different order as a vector $\mathbf{U}_{\mathrm{s}-\mathrm{s}, X Y}$, i.e. $\mathbf{U}_{\mathrm{s}-\mathrm{s}, X Y}^{l}=\left(U_{\mathrm{s}-\mathrm{s}, X Y, 1}, U_{\mathrm{s}-\mathrm{s}, X Y, 2} \ldots U_{\mathrm{s}-\mathrm{s}, X Y, 8}\right)$, where the superscript ${ }^{1}$ indicates transposition. In Appendix A, it is shown that the standard deviation of the contribution to $\Delta E_{\text {end }}$ from cutting/formation of $X Y$ pairs can be written using matrix-vector notation,

$$
\begin{aligned}
& \sigma\left[\Delta E_{\text {end }, \mathrm{s}-\mathrm{s}, X Y}\right]=\frac{\xi_{d}}{b^{2}} c_{X} c_{Y} \frac{1}{\left(1+\delta_{X Y}\right)} \\
& \left.\times \mathbf{U}_{\mathrm{s}-\mathrm{s}, X Y}^{l}\left[\mathbf{C}_{1}+\mathbf{C}_{2}\left(c_{X}+c_{Y}\right)-\mathbf{C}_{1}+2 \mathbf{C}_{2}\right) c_{X} c_{Y}\right] \mathbf{U}_{\mathrm{s}-\mathrm{s}, X Y}{ }^{\frac{1}{2}},
\end{aligned}
$$

where $c_{X}$ and $c_{Y}$ are the average concentrations of atom types $X$ and $Y$, respectively; $d$ is the width of the stacking fault ribbon; and $\mathbf{C}_{1}$ and $\mathbf{C}_{2}$ are matrices of constant coefficients, see Eq. A.15. The form above accounts for the fact that pairs $X Y$ and $Y X$ are equivalent. Thus, the total standard deviation of the solutedislocation binding energy for an alloy with $N_{T}$ atom types is the double sum

$$
\sigma\left[\Delta E_{\text {end,s-s }}\right]=\stackrel{\breve{u}}{\sum_{\substack{X, Y \geq X}}^{N_{T}}\left(\sigma\left[\Delta E_{\text {end,s-s, } X Y}\right]\right)^{2} .}
$$

Combining the two sources of energy change upon crossslip, the total standard deviation of energy changes from solutedislocation and solute-solute-interactions is

$$
\sigma\left[\Delta E_{\text {end }}\right]=\frac{\mathrm{q}}{\left(\sigma\left[\Delta E_{\text {end,s-d }}\right]\right)^{2}+\left(\sigma\left[\Delta E_{\text {end,s-s }}\right]\right)^{2}}
$$

To verify the model, we compare predictions for $\sigma\left[\Delta E_{\text {end }}\right]$ in $\mathrm{Ni}-\mathrm{Al}, \mathrm{Al}-\mathrm{Mg}$ and $\mathrm{Cu}-\mathrm{Ni}$ alloys to results from atomistic calculations reported in our previous publication [13], where the dislocation length $\zeta$ was $40 b$, the radius of the (cylindrical) configuration was $10 \overline{3} a$, and 100 (Ni-Al and Al-Mg alloys) or 120 (Cu-Ni alloys) statistical realizations were studied. For a few alloys, we have recomputed the energies using a larger radius of $13 \overline{3} a$, and we only report these new results. The Ni-Al, Al$\mathrm{Mg}$, and $\mathrm{Cu}-\mathrm{Ni}$ alloys were modeled using the Embedded Atom Method (EAM) [33] potentials in Refs. [34-36]. Note that EAM potentials are many-bodied, i.e. non-pairwise. Therefore, usage of the term "solute pairs" in this paper should not be misunderstood as indication that simple pairwise interatomic potentials were used. The solute-dislocation and solute-solute binding energies in Eq. 3 and Eq. 6 were calculated as described in Ref. [13], using the same interatomic potentials, and the average-atom approximation $[27,28]$ for the matrix atoms.

Predictions are made with increasing number of solute-solute interactions, starting from first neighbor interactions. The predicted standard deviations in energy change are shown in Fig. 3 for successive sets of neighbors up to fifth neighbors, and the atomistic simulation data from Ref. [13] is also shown. The error bars show the standard error estimate $\sigma\left[\Delta E_{\text {end }}\right] / \overline{2 n}$ [37], where $n$ is the number of realizations and normality is assumed. Additionally, histograms of the distributions are provided in Sec. S1 of the supplementary materials. In the Ni-Al alloys (Fig. 3a)), the first neighbor pairs dominate due to the very strong Al-Al near-neighbor repulsion. Further-neighbor interactions are, however, important in Al-Mg (Fig. 3b)) at higher $\mathrm{Mg}$ concentrations and in the $\mathrm{Cu}-\mathrm{Ni}$ alloys (Fig. 3c)). In $\mathrm{Cu}-\mathrm{Ni}$, the full model correctly predicts the peak in standard deviation energy at ca. 68 at.\% Ni, which is not seen if only first-nearest neighbor pairs are taken into account. The maximum absolute difference between prediction and simulation is $-0.084 \mathrm{eV}$, in $\mathrm{Al}+14$ at.\% Mg. Overall, contributions from sixth to eighth order pairs are typically less than $0.01 \mathrm{eV}$. These results demonstrate that the standard deviation in energy change due to cross-slip can be computed with high accuracy using only atomistic information on solute-dislocation and solute-solute interaction energies, which can be obtained/estimated using first-principles methods for any system of interest. See for example Refs. [31, 32, 38] for first-principles calculation of solute-dislocation binding energies. The analysis of $\sigma\left[\Delta E_{\text {end }}\right]$ is thus not limited to systems for which interatomic potentials are available; we have simply used such systems to establish the phenomena and to demonstrate the high accuracy of the theory.

\section{Random walk model}

While the correlation of the activation barrier with the endstate energy difference is powerful, its exact origin is unclear and it is valid only for segments at the critical cross-slip length. The analysis of $\Delta E_{\text {end }}$ above shows that it depends on the length $\zeta$ of the cross-slipped segment. While the correlation at $\zeta=40 \mathrm{~b}$ allows us to make contact with the simulations, and $\approx 40 \mathrm{~b}$ is the critical nucleation length for all the materials studied by simulation, nucleation at the critical length does not imply cross-slip 

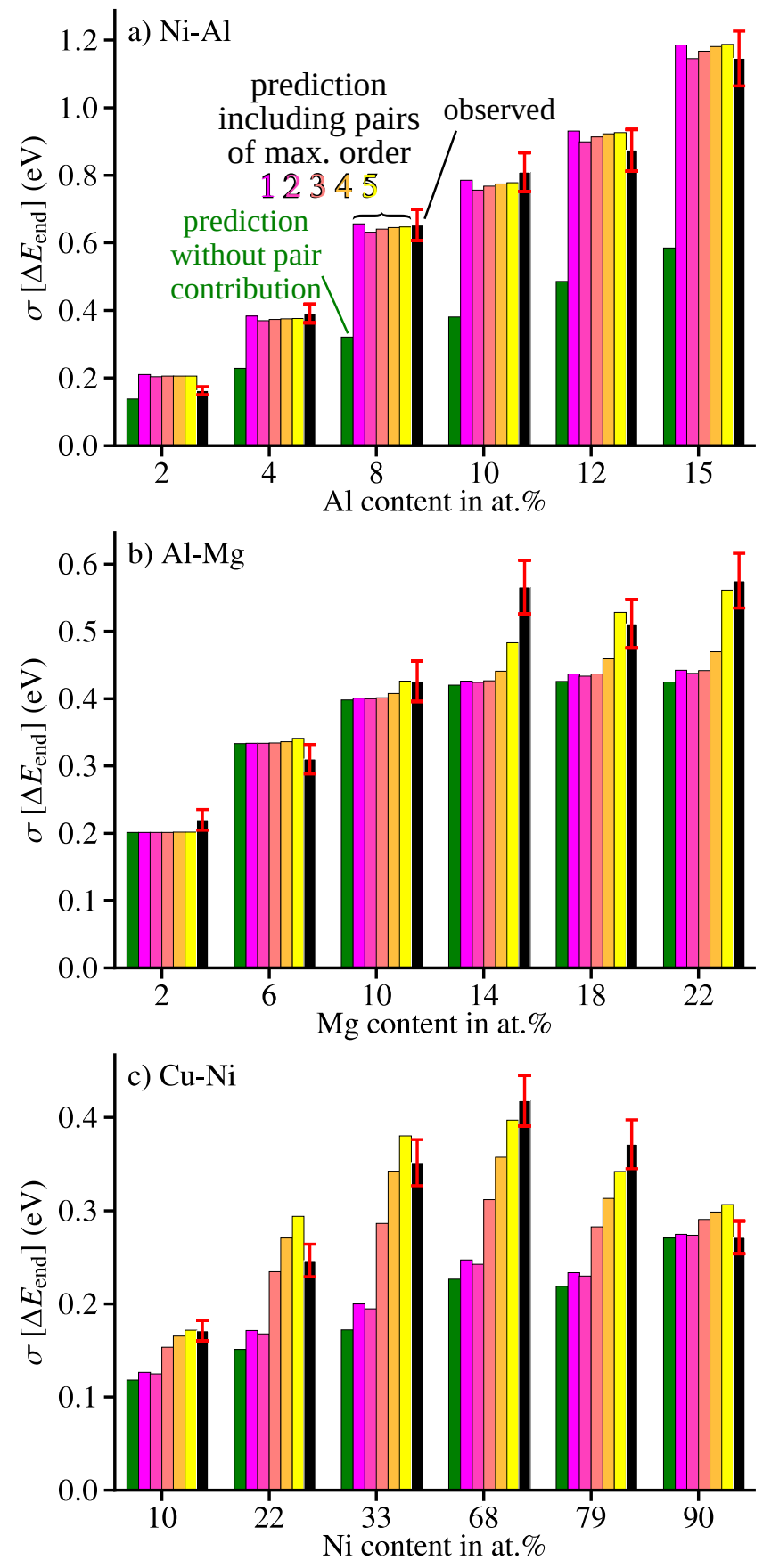

Figure 3: Standard deviation of $\Delta E_{\text {end }}$ for a $40 \mathrm{~b}$ long dislocation; note that $\Delta E_{\text {end }}$ includes solute-solute and solute-dislocation binding energy changes; colored bars: predictions made with Eq. 8; the different colors indicate the maximum order of neighbor pairs included in the calculation. Black bars: observations from atomistic calculations reported in [13] and from additional atomistic calculations described in Appendix B.1; number of realizations $n$ is 100, except in the case of $\mathrm{Cu}+22$ at. $\% \mathrm{Ni}$ and $\mathrm{Cu}+90$ at. $\% \mathrm{Ni}$, where it is 120 ; errorbars show the estimate $\sigma\left[\Delta E_{\text {end }}\right] / \overline{2 n}$ for the standard error of $\sigma\left[\Delta E_{\text {end }}\right]$ along the entire length of the typical dislocation segments in real materials $(500 b-5000 b)$. Once nucleated, the critical crossslipped segment must expand laterally, and it will encounter successive statistically-distributed solute configurations. Some of these configurations may be energetically costly (favoring the initial state). The operative cross-slip is thus the maximum barrier encountered during the expansion, which is related to the accumulated energy change plus a deterministic energy corresponding to the formation of the initial constriction. The overall process of nucleation and expansion thus suggests a randomwalk type model where each unit of lateral expansion of the cross-slipped portion has an associated random energy change. We now present such a model in detail, including the effects of Escaig and Schmid stresses that are well-established to affect the activation barrier in elemental metals.

Consider a screw dislocation with a length of $N_{b} b$. We partition the dislocation into $N_{b}$ unit segments ${ }^{3}$ of length $1 b$. We view cross-slip as a discrete process consisting of $N_{b}$ steps. On each step, one unit segment is flipped from the glide to the crossslip plane, as shown schematically in Fig. 1. Note that there is no notion of rate or time in this process. Each unit segment is either fully dissociated on the glide plane or fully dissociated on the cross-slip plane, and the current cross-slip segment consists of a contiguous set of $N_{\text {flip }}$ flipped unit segments, each of length $b$. Cross-slip can nucleate starting in any one of the $N_{b}$ segments. After the first flip, the nucleus grows by successively flipping the left or right neighboring unit segment at either end of the current cross-slipped segment. Associated with each flip is a random change in binding energy selected from the normal distribution with standard deviation $\sigma\left[\Delta E_{\text {end }}\right]$ (Eq. 8), zero mean, and unit segment length $\zeta=1 b$. The unit segment (left or right) chosen for the next flip is the unit segment with the lower random energy change, see Fig. 4. This rule reflects that the lower out of the two energy barriers is more likely to be overcome first by thermal activation. During the initial growth of the cross-slip nucleus ( $N_{\text {flip }}<N_{c}$ where $N_{c} b$ is the total length of the two constrictions), the deterministic energy cost to form a constriction must also be introduced. This energy and additional deterministic energies under stress are discussed in Sec. 3.1 and Sec. 3.2, respectively. For a given starting point, the total energy after $N_{\text {flip }}$ flips is the sum of deterministic and random contributions. The process is continued until $N_{\text {flip }}=N_{b}$, i.e. until the entire dislocation has cross-slipped. The activation energy $\Delta E_{\text {act }}$ is the maximum energy encountered in the process. The process is repeated for all $N_{b}$ possible starting points, using the same set of random energies for the segments. We thus obtain $N_{b}$ possible paths. We select the one with the lowest $\Delta E_{\text {act }}$, for the same reason that we prefer low energy steps during lateral expansion. The lowest $\Delta E_{\text {act }}$ is most likely to be overcome first by thermal activation, hence the corresponding path is the most likely operative cross-

${ }^{3}$ Note that $1 b$ is the length of a unit cell in the $h 110 i$ direction, i.e. the dislocation line direction. $1 b$ is thus the length of the shortest lattice vector with which we can measure the growth of the nucleus. As for a standard random walk, taking longer steps does not properly for statistics. One cannot execute a random walk by moving by $\pm n$ steps in each increment $(n>1)$. After $N$ steps of unit 1 , the mean squaredisplacement is $\bar{N}$; after $N$ steps of unit $n$, the mean square displacement is $n \bar{N}$, not $\overline{N n}$. 
slip path for the given set of random energies.

Due to the selection of low energy steps and paths, our process is not a standard random walk. Nevertheless, we think that the term random walk describes our process reasonably because the evolution of the cross-slip by one step length in either direction is not unlike a random walk and each step involves an additional energy chosen from a pre-determined random distribution.

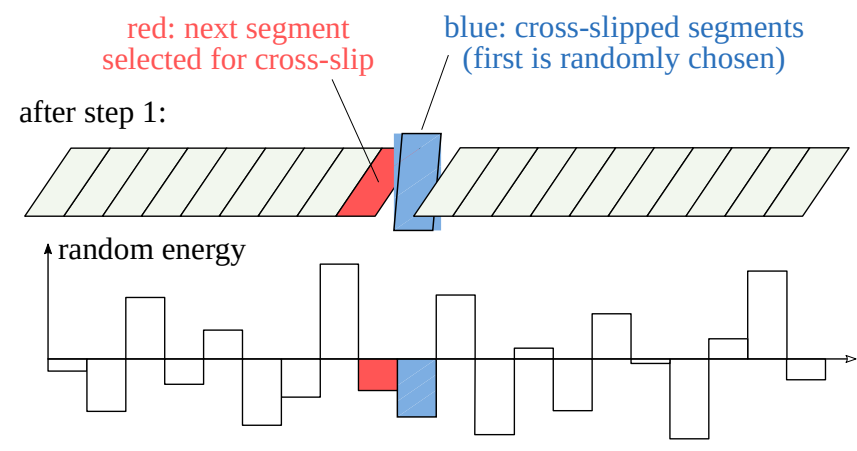

after step 2:
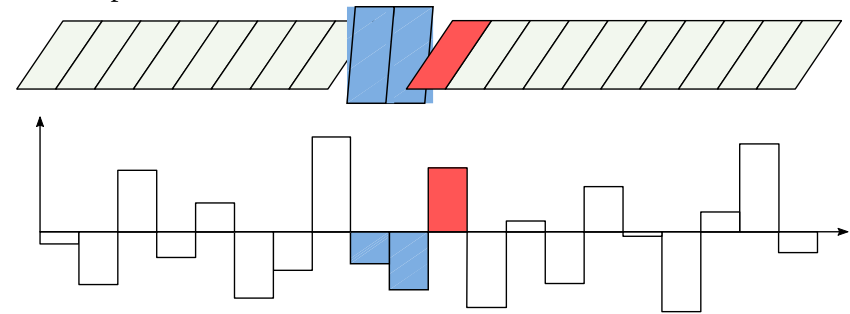

after step 3:
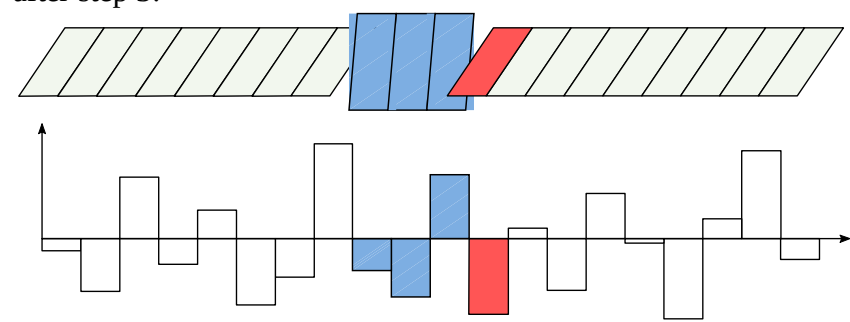

Figure 4: Step selection in the random walk; cross-slip can nucleate in any of the $1 b$ segments; subsequently, however, low-energy steps are preferred

\subsection{Constriction energy}

In pure metals at zero stress, the activation energy of FEcross-slip is approximately the formation energy of two point constrictions, i.e. $\Delta E_{\text {act,avg }} \approx 2 E_{c}$ [39]. This total energy is distributed over a physical length $N_{c} b$; that is, the full two-pointconstriction energy builds gradually over some physical length, as seen in directly atomistic models of the transition path. In this section, we present a simple model of the constriction energy as a function of the number of cross-slipped segments. We note at the outset that $\Delta E_{\text {act,avg }}$ and $N_{c}$ need to be known.

The first unit segment on the cross-slip plane cannot nucleate without the formation of a full constriction on the glide plane. Thus, there is an additional energy cost for the first flip of $E_{c}=\Delta E_{\text {act,avg }} / 2$. The two half-constrictions on the cross-slip plane then grow as the cross-slipped segment becomes longer. At a length $N_{c} b$, the half-constrictions are well-separated and the total energy cost of forming the constrictions has been accumulated. To model this, we assume that the additional constriction energy per flip decreases linearly as the segment length grows from $b$ to $N_{c} b$. The total energy thus increases quadratically. At flip $i$ of the nucleation process, the total deterministic energy is thus given as

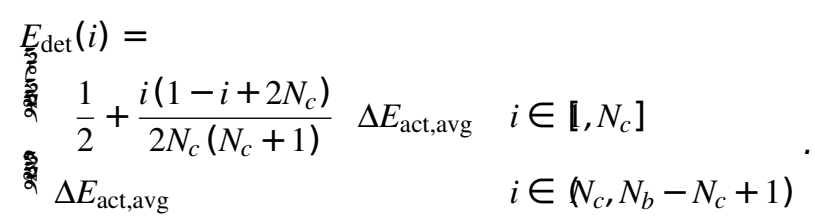

For simulations, we use periodic boundary conditions to eliminate end effects. Thus, when the cross-slip process is nearly complete, the constrictions should annihilate, and the energy regained. Thus, the deterministic energy is modified as follows for $i$ in the range $\left[N_{b}-N_{c}+1, N_{b}\right)$ :

$$
E_{\text {det }}(i)=\Delta E_{\text {act,avg }} 1-\frac{\left(i-N_{b}+N_{c}\right)\left(1+i-N_{b}+N_{c}\right)}{2 N_{c}\left(N_{c}+1\right)},
$$

and $E_{\operatorname{det}}\left(N_{b}\right)=0$.

Fig. 5 shows the energy vs. cross-slipped length from an atomistic transition path calculation for $\mathrm{Ni}+15$ at.\% $\mathrm{Al}$ as compared to the deterministic energy of Eq. 9 using the atomistic activation energy and $N_{c}=10$. The length of the cross-slipped segment in the atomistic calculation was measured using the dislocation analysis (DXA) algorithm implemented in Ovito [26]. Atomistically, the constriction forms at zero length, but this energy must be assigned to the first unit segment of length $b$. The use of $\Delta E_{\text {act,avg }} / 2$ for the first flip underestimates the atomistic energy by approximately $0.19 \mathrm{eV}$ on the first step. Larger errors are seen in the region of constriction annihilation. This may explain later results in which there are small $(<0.1 \mathrm{eV})$ differences between the median values of activation energy distributions from atomistic and random walk calculations, but the model of annihilation is only of importance for comparing to the simulation results at short total lengths $\left(N_{b}=40\right)$. Overall, the model for constriction formation and annihilation is in good qualitative agreement with the atomistic simulation, and is fully satisfactory for the purposes of this paper.

\subsection{Incorporation of Escaig and Schmid stresses}

Escaig stresses that lower the energy of the final state relative to the initial state will facilitate cross-slip. Net Schmid stresses acting on the cross-slip plane will also facilitate cross-slip. Both types of stresses will also help to overcome very high activation barriers that can arise in the random walk under zero stress. The work done by such external stresses is deterministic and hence can be included in the random walk model by modifying the deterministic energy contribution in Eq. 9. 


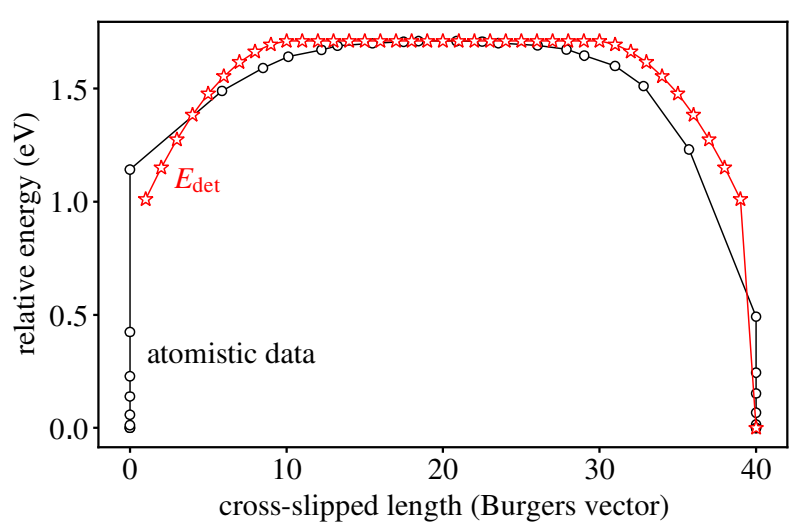

Figure 5: Relative energy as a function of length of the cross-slipped segment from an atomistic transition path calculation with average $\mathrm{Ni}+15$ at.\% $\mathrm{Al}$ (black circles) and the corresponding approximate profile $E_{\text {det }}$ used in random walk calculations (red stars); the first few points at zero length in the atomistic profile correspond to formation of the constriction on the glide plane.

We first address Escaig stresses, which couple to the edge components of the partial dislocations of the dissociated screw dislocation in FCC materials. These stresses change the width of the partial separation. Let $\tau_{\text {glide }}^{\text {Esc }}$ and $\tau_{\text {cross }}^{\text {Esc }}$ be the Escaig stresses on the glide and cross-slip plane, respectively. These stress have two effects. First, they do work on the system when the splitting width changes from its equilibrium value on the glide plane to its equilibrium value on the cross-slip plane. We assume that these equilibrium widths are the same for all $1 b$ segments, hence the same amount of work $E_{\mathrm{det}}^{\text {Esc }}$ is done on each segment, and the total work after cross-slip of $i$ segments is $i E_{\mathrm{det}}^{\mathrm{Esc}} . E_{\mathrm{det}}^{\mathrm{Esc}}$ can be computed in atomistic simulations, but models using anisotropic elasticity are more general. When comparing with atomistic simulations, the atomistic result for $E_{\mathrm{det}}^{\text {Esc }}$ was used, see Appendix B.4. In other cases, an anisotropic-elastic estimate ${ }^{4}$ was used,

$$
E_{\mathrm{det}}^{\mathrm{Esc}}=-\frac{b^{3}}{8 \pi} \quad K_{s}-\frac{K_{e}}{3} \log \frac{\chi_{\mathrm{ff}, g}}{\chi_{\mathrm{ff}, c}},
$$

where $K_{e}$ and $K_{s}$ are the energy prefactors of the edge and screw dislocation, respectively, [41, Equ. 13.149b] and $\chi_{\mathrm{ff}, g}$ and $\chi_{\mathrm{ff}, c}$ are the effective stacking fault energies on the glide and crossslip plane,

$$
\chi_{\mathrm{ff}, g}=\chi_{\mathrm{f}}+\frac{b \tau_{\mathrm{gdide}}^{\text {Esc }}}{2 \overline{3}}, \quad \chi_{\mathrm{fff}, c}=\chi_{\mathrm{f}}-\frac{b \tau_{\mathrm{foss}}^{\text {Esc }}}{2 \overline{3}} .
$$

Second, the Escaig stresses change the constriction energy. According to Püschl and Schoeck [6, 39] $E_{c} \propto \log (2 d / b) d / b$, thus we scale the energy of the constriction on the glide plane by the ratio $\left(\log \left(2 d^{*} / b\right) / \log (2 d / b)\right)\left(d^{*} / d\right)$, where $d^{*}$ is the stressed equilibrium dissociation width. Using the anisotropic-elastic solution for $d^{*}$ [41, pp. 361], this ratio can be written as

$$
f_{\mathrm{c}}=\frac{\chi_{\mathrm{ff}}}{\chi_{\mathrm{ff}, g}} \frac{\log \left(K_{s}-K_{e} / 3\right) b / 4 \pi \gamma_{\mathrm{eff}, g}}{\log \left(\left(K_{s}-K_{e} / 3\right) b /\left(4 \pi \gamma_{\mathrm{sf}}\right)\right)} .
$$

\footnotetext{
${ }^{4}$ Equ. 5.7 in Ref. [ 40] with $d_{M}=d$ and splitting widths from anisotropic elasticity [41, Equ. 13.149b]
}

Accounting for the effect of $\tau_{\text {cross }}^{\text {Esc }}$ on the constriction on the crossslip plane is more complicated, because the stress also changes the constriction size and hence $N_{c}$. Thus, we neglect this energy contribution. Nevertheless, we obtain good agreement between predicted activation energies and atomistic data, see Appendix C.

Net Schmid stresses on the cross-slip plane couple to the screw parts of the partial dislocations, which causes bowing out of the cross-slipped segment between the two constrictions. We consider two energy changes associated with the bow-out. First of all, it causes an increase of the total dislocation length, which, in turn, increases the energy of the configuration. This cost is represented by a line tension parameter $T$. Second, the external stress does work over the swept area $A$. If the cross-slipped segment is sufficiently long, the latter dominates and the energy of the configuration is further reduced when the bow-out grows. In this way, a bow-out can drive cross-slip. We assume that the bow-out takes the shape of a circular arc with radius $R$ and arc-length $s$. Within isotropic linear elasticity, the total energy of bowing out can be described analytically [42]. For a size $i b$ of cross-slipped segment, the total energy due to bowout is

$$
\begin{aligned}
E_{\mathrm{det}}^{\mathrm{Sch}}(i) & =T s(i)-\tau_{\mathrm{cross}}^{\mathrm{Sch}} b A(i), \\
\text { where } & \\
s(i) & =2 R \sin ^{-1} \frac{i b}{2 R} \mathrm{~s}-i b, \\
A(i) & =R^{2} \sin ^{-1} \frac{i b}{2 R} 1-\frac{i b}{2 R}{ }^{2}, \\
R & =\frac{T}{\tau_{\text {cross }}^{\mathrm{Sch}} b}
\end{aligned}
$$

and $T$ is an effective dislocation line tension representing the cost of creating additional dislocation line length.

The value of $T$ is guided by the work of Kang et al. [8], who compared the stress dependence of the cross-slip activation energy in the Friedel-Escaig model and in atomistic simulations. They suggested $T=\alpha \mu b^{2} / 2$, where [43]

$$
\mu \equiv K_{s}=\frac{r}{\left(C_{11}-C_{12}\right) C_{44}},
$$

and $\alpha=0.45$. However, the FE-model overestimated the activation energy. We thus use a rather smaller value of $T=$ $0.075 \mu b^{2} / 2$, which yields activation energies in our model that are much closer to the atomistic results of Kang et al. than the predictions of the FE-model. Additional random energy fluctuations during slip from the straight to the bowed-out configuration due to solute-dislocation interactions (essentially solute strengthening) are neglected.

In total, in the presence of Escaig and/or cross-slip Schmid stress, the deterministic energy profile for the random walk 
model is

$$
\begin{aligned}
& E_{\text {det }}(i)= \\
& \text { 商 } \left.\frac{f_{\mathrm{c}}}{2}+\frac{i\left(1-i+2 N_{c}\right)}{2 N_{c}\left(N_{c}+1\right)} \Delta E_{\mathrm{act}, \mathrm{avg}}+i E_{\mathrm{det}}^{\mathrm{Esc}}+E_{\mathrm{det}}^{\mathrm{Sch}}(i) \quad i \in \llbracket, N_{c}\right] \\
& \text { क्ष } \frac{f_{\mathrm{c}}+1}{2} \Delta E_{\mathrm{act}, \mathrm{avg}}+i E_{\mathrm{det}}^{\mathrm{Esc}}+E_{\mathrm{det}}^{\mathrm{Sch}}(i) \quad i>N_{c}
\end{aligned}
$$

Under periodic boundary conditions for comparison to atomistic simulations, the constriction is annihilated in the manner used at zero stress; this is only relevant for pure Escaig stresses.

In summary, a dislocation line of length $N_{b}$ is first envisioned to consist of a set of $1 b$ segments. Each $1 b$ segment has an energy change for cross-slip, due to the local random arrangement of solutes, that is chosen from a normal distribution with mean zero and standard deviation given by Eq. 8. The cross-slip starts by nucleation of a $1 b$ segment at some position along the line. The cross-slip segment grows by the sequential cross-slipping of the individual $1 b$ segments at either end of the growing crossslipped segment. The energy change upon cross-slip of each $1 b$ segment involves the pre-assigned random energy plus a deterministic energy. The deterministic energy accounts for the initial constriction and separation of the two constrictions occurring over the first $N_{c}$ steps (Eq. 9 and Fig. 5). In the presence of applied Escaig and/or Schmid bow-out stresses, there are additional deterministic energies associated for every segment (Eqs. 11, 13 and 14). The cross-slip energy barrier is then the maximum energy along the path starting from the initially-chosen $1 b$ segment and ending at the total length $N_{b}$. All $N_{b}$ possible $1 b$ segments are then considered as the starting point for cross-slip. The lowest energy barrier among all starting points is the energy barrier for the specific realization of the $N_{b}$ pre-assigned random energies.

\section{Validation against atomistic simulations}

Here, we compare predictions of the random walk model against the full atomistic transition state simulations at $N_{b}=40$ using periodic boundary conditions, for both zero stress and applied Escaig stresses. The results demonstrate that the random walk model captures all major features of the simulations with very good quantitative accuracy, justifying its application to long dislocation lines $\left(N_{b} \quad 40\right)$ in the next section of this paper.

Materials studied are the $\mathrm{Ni}-\mathrm{Al}, \mathrm{Al}-\mathrm{Mg}$, and $\mathrm{Cu}-\mathrm{Ni}$ alloys modeled using the EAM [33] potentials in Refs. [34-36] and studied previously [13], These materials cover a wide range of scenarios. In $\mathrm{Ni}-\mathrm{Al}$, the $\mathrm{Al}-\mathrm{Al}$ pair energy is high and dominates $\Delta E_{\text {end }}$. In Al-Mg, $\Delta E_{\text {end,s-d }}$ provides the main contribution to $\Delta E_{\text {end }}$ at low to medium concentrations. In $\mathrm{Cu}-\mathrm{Ni}$, high concentrations are considered and beyond near-neighbor solute-solute interactions are important (see Figure Fig. 3). The quantities $\Delta E_{\text {act,avg }}$ and the standard deviaton of the random energy per unit step for each material are listed in Tab. 1.

\subsection{Zero stress}

At zero Escaig stresses, a total of 100 realizations were used to generate the atomistic results and a total of 10000 realizations
Table 1: Average-alloy cross-slip activation energy $\Delta E_{\text {act,avg and standard }}$ deviation of the random energy per $1 b$ step, as calculated with Eq. 8

\begin{tabular}{ccc}
\hline alloy & $\Delta E_{\text {act,avg }}(\mathrm{eV})$ & $\sigma\left[\Delta E_{\text {end }}\right] \xi=1 b$ \\
\hline $\mathrm{Ni}+02 \% \mathrm{eV})$ \\
$\mathrm{Ni}+15 \% \mathrm{Al}$ & 1.73 & 0.033 \\
$\mathrm{Al}+02 \% \mathrm{Mg}$ & 0.73 & 0.189 \\
$\mathrm{Al}+06 \% \mathrm{Mg}$ & 0.69 & 0.032 \\
$\mathrm{Al}+22 \% \mathrm{Mg}$ & 0.63 & 0.054 \\
$\mathrm{Cu}+22 \% \mathrm{Ni}$ & 1.65 & 0.088 \\
$\mathrm{Cu}+33 \% \mathrm{Ni}$ & 1.64 & 0.047 \\
$\mathrm{Cu}+79 \% \mathrm{Ni}$ & 1.51 & 0.062 \\
\hline
\end{tabular}

were used in the (much faster) random walk calculations. Fig. 6 shows a box plot of the activation energy distributions at zero stress for a number of alloys. The line in each box indicates the median, while the lower and upper edges show the first and third quartile, respectively. Histograms of the distributions are presented in Sec. S2 in the supplementary materials.

Atomistic and random walk results are typically similarly distributed. The difference in median values is always less than $0.07 \mathrm{eV}$ (in $\mathrm{Ni}+15$ at. $\% \mathrm{Al}$ ) and the largest relative difference is $12 \%$ (in $\mathrm{Al}+22$ at.\% $\mathrm{Mg}$ ). The largest relative difference in variance is $78 \%$ (in $\mathrm{Ni}+02$ at.\% $\mathrm{Al}$ ) but the actual difference is very small (0.007 eV higher than the atomistic value). The similitude of the distributions is assessed by the two-sided KolmogorovSmirnov (K-S) [44, 45] statistic. We test the Null hypothesis [46] that both the atomistic and random walk samples are drawn from the same distribution. It can only be rejected at significance level 0.05 in the case of $\mathrm{Al}+02$ at.\% $\mathrm{Mg}$ and $\mathrm{Ni}+02$ at.\% $\mathrm{Mg}$. If the random walk samples are shifted to eliminate the difference in median values, the Null hypothesis cannot be rejected in any case. Thus, the random walk model typically yields distributions very similar to those obtained from the atomistic calculations.

Small differences in median values are likely caused by deviations of $E_{\mathrm{det}}$ from the true deterministic energy profile. For example, in the case of $\mathrm{Ni}+02$ at.\% $\mathrm{Al}, E_{\text {det }}$ overestimates the true energy at the end of constriction formation and during annihilation, see Fig. 5. However, these are also the regions where the total energy, i.e. the sum of deterministic and random energies, frequently achieves its maximum value, see Fig. 7. Therefore, random walk calculations tend to yield higher activation energies than atomistic calculations in the case of $\mathrm{Ni}+02$ at.\% $\mathrm{Al}$. For completeness, we note that a similar distribution of the location of the maximum energy is obtained for other alloys - see the results for $\mathrm{Ni}+15$ at.\% $\mathrm{Al}$ in Fig. 7 - and longer dislocations. In general, the maximum energy frequently occurs near the beginning or end of the walk.

Furthermore, the random walk model predicts the correlation between the activation energy $\Delta E_{\text {act }}$ and the end-state difference $\Delta E_{\text {end }}$ that was empirically found in the atomistic simulations at length $40 b$ [13]. For example, Fig. 2 shows $\Delta E_{\text {act }}$ versus $\Delta E_{\text {end }}$ from the atomistic and random walk calculations for $\mathrm{Ni}+15 \% \mathrm{Al}$. Linear regression of both data sets yields slopes of 0.5 and 0.54 , respectively. Slopes of $0.53-0.54$ are obtained from the random walk calculations in other materials. Therefore, 


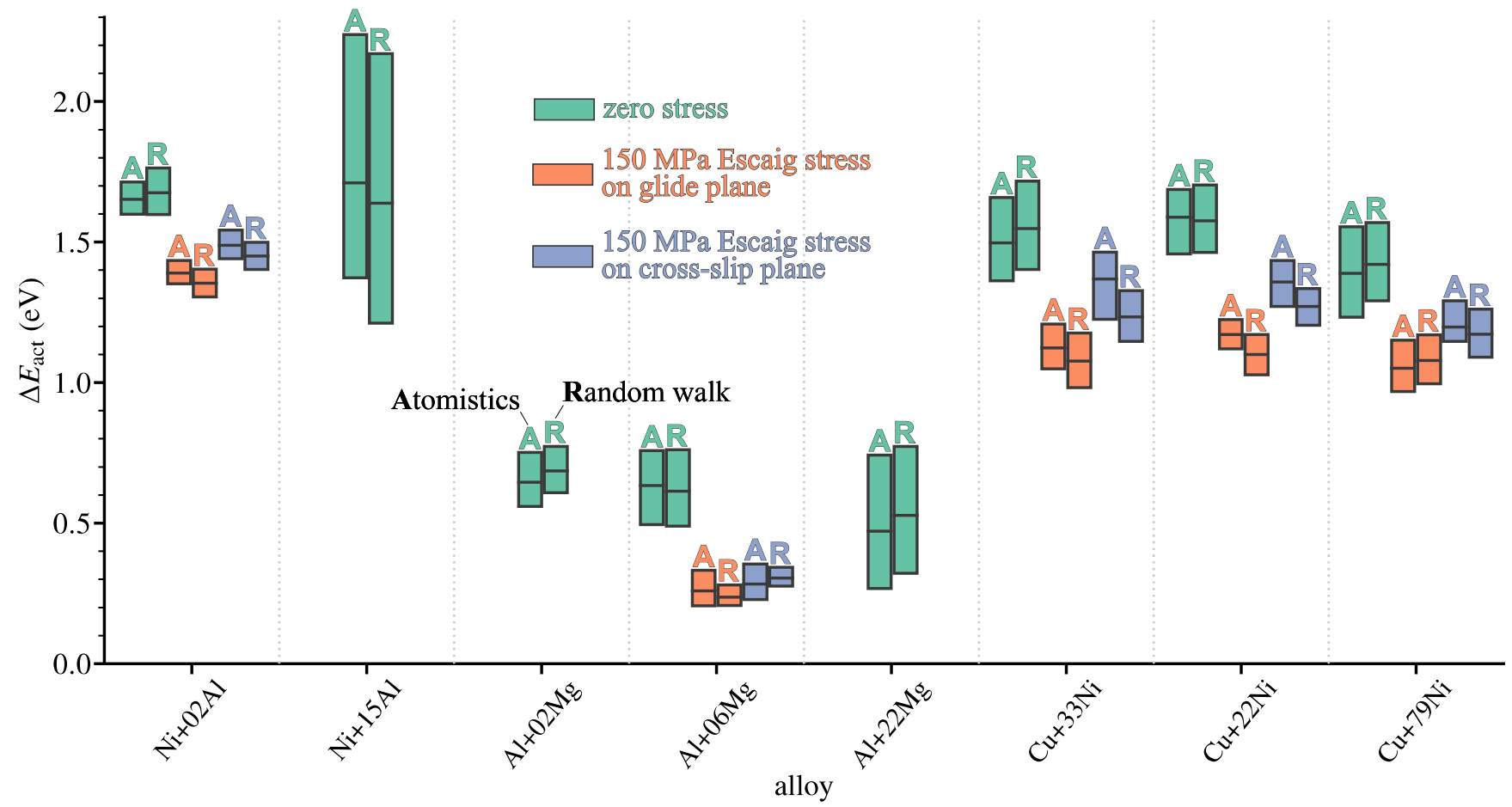

Figure 6: Distributions of cross-slip activation energies in solid solution alloys for $N_{b}=40$ with periodic boundary conditions, from atomistic (A) and random walk (R) simulations; the upper and lower edge of each box represent the third and second quartile of the data, i.e. 50\% of all values lie in this range; the horizontal lines indicate the median. The number of realizations in atomistic calculations was 100, except in the case of $\mathrm{Cu}+22$ at. $\% \mathrm{Ni}$ and $\mathrm{Cu}+79$ at. $\% \mathrm{Ni}$ at finite stress where only 50 realizations were used; 10000 random walk realizations are used in all cases; for histograms of the distributions see Sec. S2 in the supplementary materials.

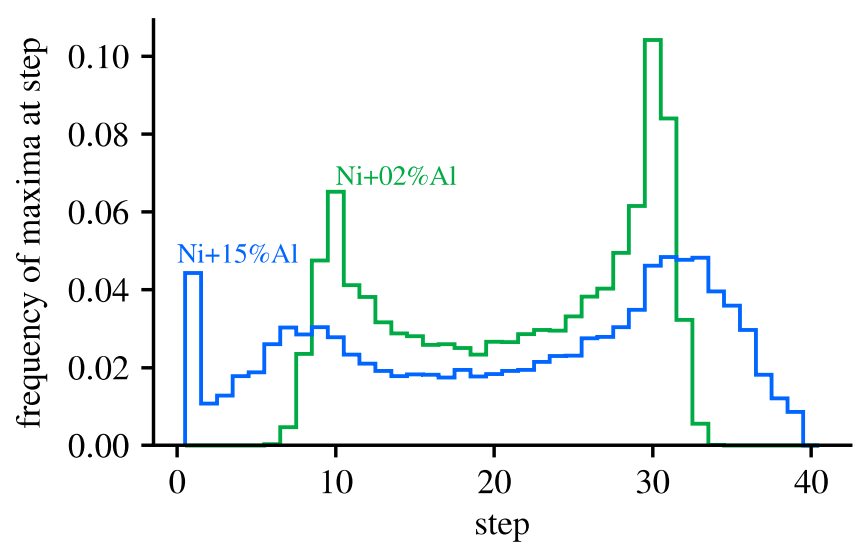

Figure 7: Frequency with which the total energy achieves its maximum value $\Delta E_{\text {act }}$ at a particular step in random walk simulations; note that the dislocation length is $40 \mathrm{~b}$ here; $\Delta E_{\text {act }}$ frequently occurs near the beginning or the end of the walk; data from calculations with $\mathrm{Ni}+02$ at. $\% \mathrm{Al}$ and $\mathrm{Ni}+15$ at. $\% \mathrm{Al}(10000$ realizations per alloy); the same trend is seen for other alloys and dislocation lengths the random walk model, with only the average barrier height and solute-solute and solute-dislocation interaction energies as inputs, reproduces a major empirical feature of the atomistic simulations.

\subsection{Non-zero Escaig stresses}

Using the same atomistic methods as in our previous work, transition state calculations were carried out for Escaig stresses of $\tau_{\text {glide }}^{\text {Esc }}=150 \mathrm{MPa}$ and $\tau_{\text {cross }}^{\text {Esc }}=150 \mathrm{MPa}$ for several alloys. For $\mathrm{Cu}+22$ at. $\% \mathrm{Ni}$ and $\mathrm{Cu}+79$ at.\% Ni, only 50 realizations were simulated. In applying the random walk model, the value

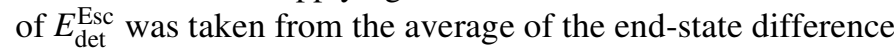
obtained in the atomistic simulations. Box plots of the results are shown in Fig. 6. For histograms of the data see Sec. S2 in the supplementary materials. The difference in median values is typically small, with a maximum relative difference of $10 \%$ $(0.14 \mathrm{eV})$ in $\mathrm{Cu}+33$ at.\% Ni with stress on the cross-slip plane. The widths of the distributions are typically similar. However, in $\mathrm{Al}+06$ at. $\% \mathrm{Mg}$ there is a comparatively large relative difference in the variances, $-68 \%$ and $-78 \%$ for stresses on the glide and cross-slip plane, respectively, but only small absolute differences $(-0.008 \mathrm{eV}$ and $-0.012 \mathrm{eV}$ ). The K-S statistical analysis shows that the Null hypothesis that the samples are drawn from the same continuous distribution can be rejected at significance 0.05 for all alloys and stresses except in the case of $\mathrm{Cu}+79$ at.\% Ni. However, if the difference in median values is subtracted, then the hypothesis can only be rejected in the case of $\mathrm{Al}+06$ at.\% Mg. Thus, most of the error comes from the 
difference in median values, which is likely a consequence of errors in the deterministic energy profile, as discussed in Sec. 4.

\section{Cross-slip of long dislocations}

The dislocation length of $40 \mathrm{~b}$, which we considered in the previous section, is a typical length for cross-slip nucleation in atomistic calculations with elemental metals [13]. The close agreement between random walk calculations and atomistic calculations at this length scale indicates that the random walk model correctly samples the random energy fluctuations during cross-slip. Thus, we can use the random walk model to study full cross-slip of long dislocations, with lengths up to $1000 b$, corresponding to the typical length of dislocations in a deformed metal with a dislocation density of $10^{13} \mathrm{~m}^{-2}$. Simulations were performed at lengths $N_{b}=40,100,200,500,1000$ with no periodic boundary conditions, so the constrictions were not annihilated at the end of the process, and results effectively corresponding to cross-slip of a slightly longer segment or a segment pinned by obstacles.

\subsection{Zero stress}

For zero applied stresses, Fig. 8 shows box plots of the distributions of $\Delta E_{\text {act }}$ for lengths between $40 \mathrm{~b}$ and $1000 \mathrm{~b}$. For histograms of the data see Sec. S3 in the supplementary materials. A clear trend is seen: with increasing length, the frequency of high activation energies increases. $F$ or the third quartile, we observe a trend $\Delta E_{\text {act,avg }}+\sigma\left[\Delta E_{\text {end }}\right] x \overline{N_{b} / 2}$ with $x=1.2$. The scaling of higher percentile levels follows the same form, but with larger $x$. On the other hand, the lower tail of the distribution does not change significantly. The decrease of the first quartile is much less pronounced than the increase of the third quartile. Accordingly, the median values increase by several tenths of an electron volt, in the case of $\mathrm{Ni}+15$ at.\% Al even by more than one electron volt. The minimum barrier, however, barely changes. The increasing frequency of high activation energies with increasing dislocation length arises naturally through statistics. Note that there is a correlation between the value of $\Delta E_{\text {act }}$ and the step on which it is realized. At some point, the cross-slipping dislocation must overcome all the barriers along its entire length. However, the random walk process enables the dislocation to defer encountering the highest barriers until near the very end of the process. Thus, the high activation barriers occur predominantly in the last few steps. Fig. D20 in Appendix $D$ shows an example of the correlation between the step at which $\Delta E_{\text {act }}$ is realized and the value of $\Delta E_{\text {act }}$ for $\mathrm{Cu}+33$ at. $\% \mathrm{Ni}$ and $N_{b}=200$. The consequence of the increasing frequency of high activation energies is that cross-slip of long dislocations is much harder and less frequent in solid solutions. However, this result pertains only to zero stress. As shown next, the high barriers are easily overcome by fairly modest applied stresses, leading to much lower activation energies.

\subsection{Non-zero stress}

Schmid and Escaig stresses in the range 1-200 MPa were applied to dislocations with length $N_{b}=500$. The constrictions

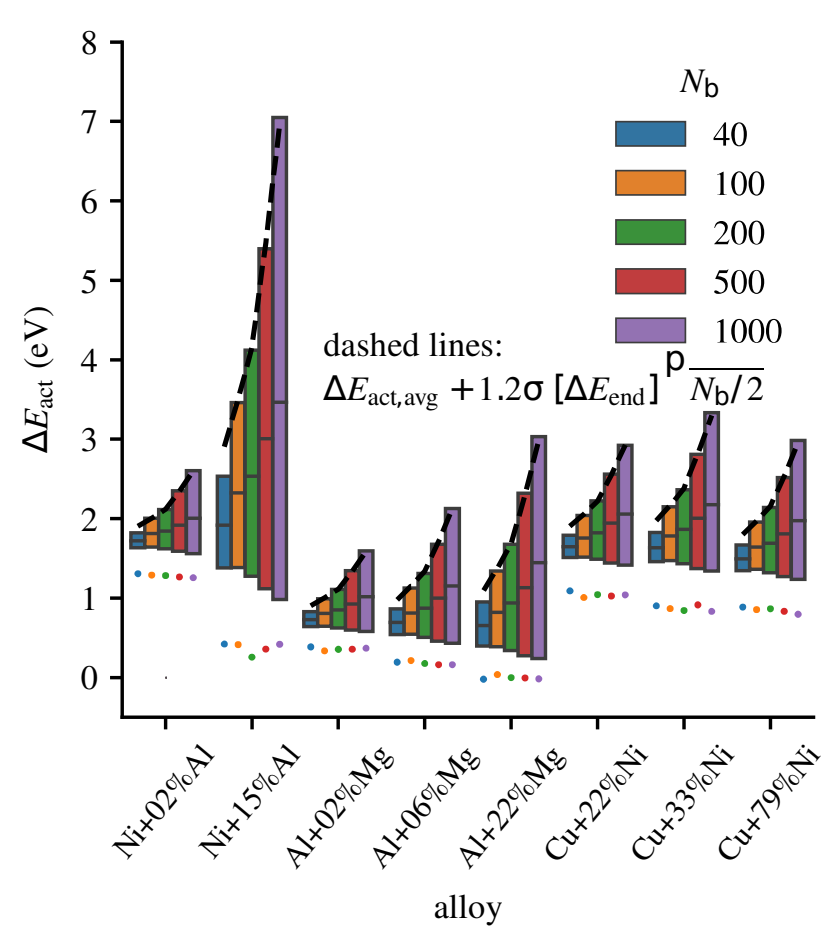

Figure 8: Distributions of activation energies for different alloys and dislocation lengths $N_{b}$; the upper and lower edge of each box represent the third and second quartile of the data, i.e. $50 \%$ of all values lie in this range; horizontal lines indicates the median; the dot marks the minimum value; for histograms of the distributions see Sec. S3 in the supplementary materials.

were not annihilated at the end of the process and no periodic boundary conditions were used. In contrast to the calculations with short segments in Sec. 4.2, we did not use the atomistic value for $E_{\mathrm{det}}^{\text {Esc }}$, but rather the elastic estimate according to Eq. 11.

Fig. 9 shows energy distributions for $\mathrm{Cu}+33$ at.\% Ni. The other alloys exhibit similar behavior, see Fig. 10. Applied stresses tend to eliminate the high energy barriers because the high $\Delta E_{\text {act }}$ tend to occur near the end of the random walk, where the cross-slip segment length is very long such that the total work done by the applied stress is then also large. The work done is then more than sufficient to overcome these large barriers. Under stress, the distribution of barriers then collapses toward the lower values of $\Delta E_{\text {act }}$, which occur at short cross-slip lengths and which are less strongly affected by the applied stress. A Schmid stress on the cross-slip plane is particularly effective, with the third quartile being reduced by $1.1 \mathrm{eV}$ upon increasing the Schmid stress from $1 \mathrm{MPa}$ to $5 \mathrm{MPa}$. Such low Schmid stress could easily be reached in real materials due to long range stresses and stress fluctuations caused by the local dislocation network. Escaig stresses, by comparison, are less effective in that higher stresses in the range of $25-50 \mathrm{MPa}$ are required to eliminate the high activation barriers. Nonetheless, such stress levels remain moderate. Note that the observed stress effects are more complicated than in elastic or atomistic models for pure metals. Usually, $\tau_{\text {glide }}^{\text {Esc }}$ is expected to be the most effective stress for reducing the activation energy while $\tau_{\text {cross }}^{\text {Sch }}$ is supposed to be least effective [8]. Fig. 9 shows that this is only true at higher stresses. 


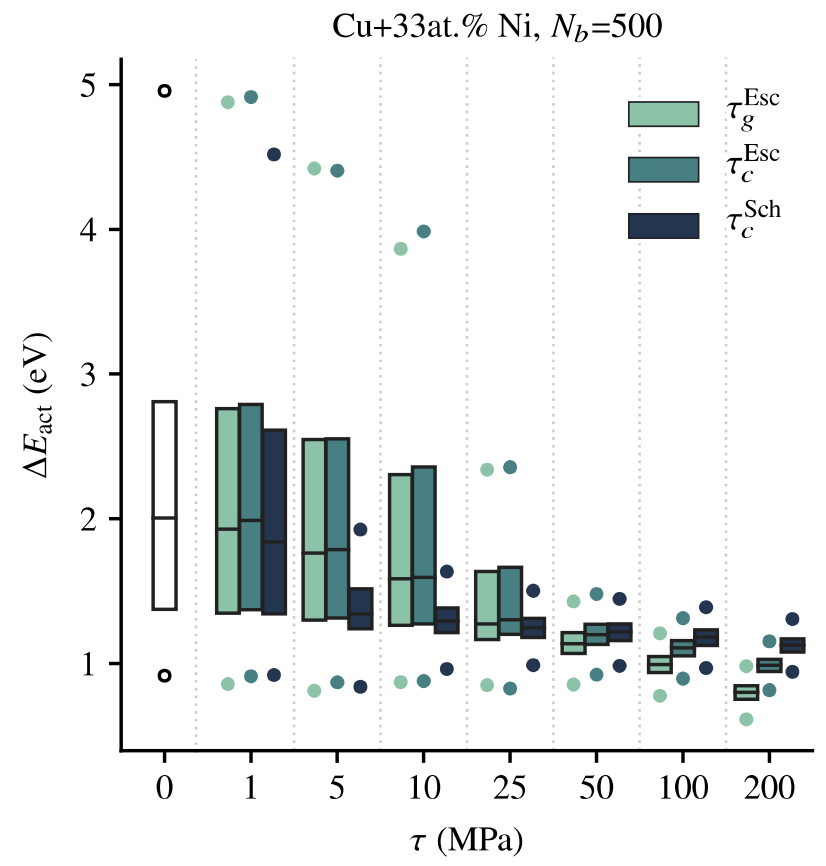

Figure 9: Activation energy distributions in $\mathrm{Cu}+33$ at. $\%$ Ni with $N_{b}=500$ for different Escaig and Schmid stresses; the upper and lower edge of each box represent the third and second quartile of the data, i.e. $50 \%$ of all values lie in this range; the horizontal lines indicate the median; the dots mark the minimum and maximum value of each dataset

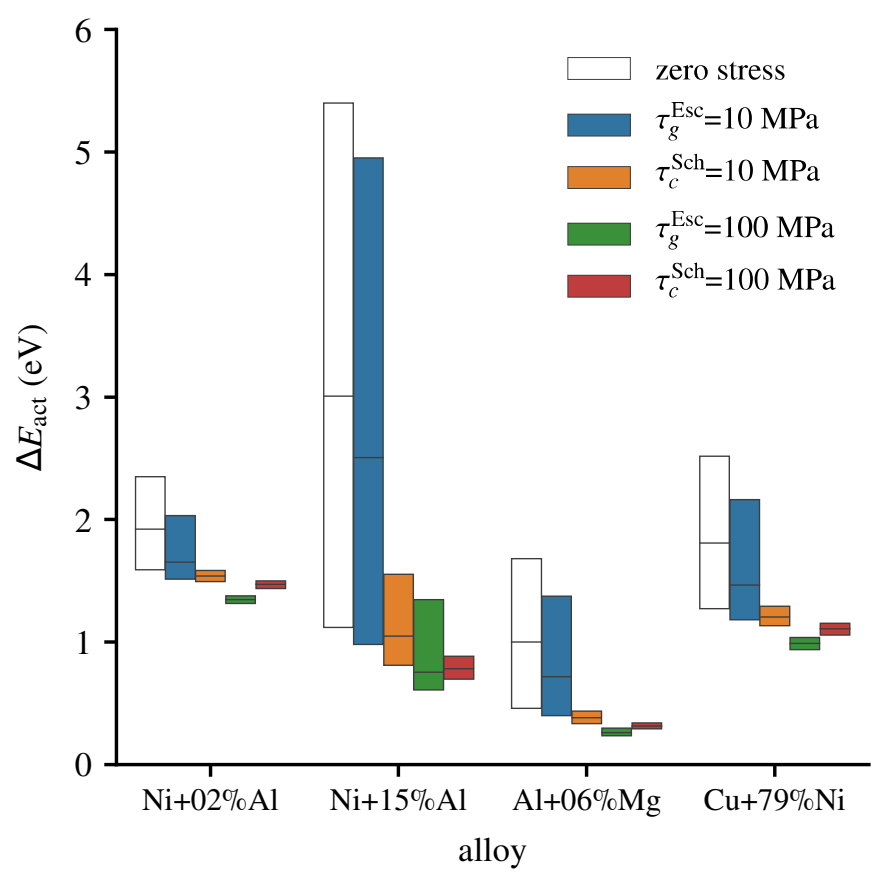

Figure 10: Activation energy distributions in solid solution alloys for $N_{b}=500$ and high or low Escaig and Schmid stresses; the upper and lower edge of each box represent the third and second quartile of the data, i.e. $50 \%$ of all values lie in this range; the horizontal lines indicate the median;
The characteristic stress required to eliminate high activation energies can be estimated as follows. As noted above, the maximum barrier is unlikely to occur at the end of the random walk if the magnitude of work performed by the external stress after $N_{b}$ steps is likely to exceed the accumulated random energy contributions. As shown in Fig. 8, a majority of the activatipn energies have an energy of less than $\Delta E_{\text {act,avg }}+\sigma\left[\Delta E_{\text {end }}\right] 1.2 \overline{N_{b} / 2}$. Thus, the work should exceed $\sigma\left[\Delta E_{\text {end }}\right] 1.2 \overline{N_{b} / 2}$. The work is represented by three terms in Eq. 16. The scaling factor $f_{\mathrm{c}}$, which accounts for the fact that a stress $\tau_{\text {glide }}^{\text {Esc }}$ changes the energy of the constriction on the glide plane, can be ignored. It only contributes an offset to the total energy and thus has no influence on the position of the maximum. The remaining work by Escaig and Schmid stresses is $i E_{\text {det }}^{\text {Esc }}\left(\tau_{\text {glide }}^{\text {Esc }}, \tau_{\text {cross }}^{\text {Esc }}\right)+E_{\text {det }}^{\text {Sch }}\left(\tau_{\text {cross }}^{\text {Sch }}, i\right)$. Setting $i=N_{b}$ and requiring that the work equals $\sigma\left[\Delta E_{\text {end }}\right] 1.2 \mathrm{p} \overline{N_{b} / 2}$, we obtain the conditon

$N_{b} E_{\text {det }}^{\text {Esc }}\left(\tau_{\text {glide }}^{\text {Esc }}, \tau_{\text {cross }}^{\text {Esc }}\right)+E_{\text {det }}^{\mathrm{Sch}}\left(\tau_{\text {cross }}^{\mathrm{Sch}}, N_{b}\right)+\sigma\left[\Delta E_{\text {end }}\right] 1.2^{\mathrm{p}} \overline{N_{b} / 2}=0$.

Assuming pure Escaig and Schmid stresses, one obtains characteristic stresses of $\tau_{\text {glide }}^{\text {Esc }}=21.3 \mathrm{MPa}, \tau_{\text {cross }}^{\text {Esc }}=20.7 \mathrm{MPa}$, and $\tau_{\text {cross }}^{\text {Sch }}=2.1 \mathrm{MPa}$ for $\mathrm{Cu}+33$ at. $\% \mathrm{Ni}$ at $N_{b}=500$, consistent with the results in Fig. 9.

In summary, moderate applied stresses that act to facilitate cross-slip are sufficient to eliminate high barriers and drive the entire distribution of activation barriers to much lower values than the mean value at short lengths.

\subsection{Weakest-link scaling}

The previous observations motivate us to treat cross-slip under stress as a weakest-link problem. At sufficiently large stress, most maxima occur early in the random walk. Thus, the activation energy distribution at a long length $N_{b}$ can be inferred from the corresponding distribution for a much shorter length $N_{\text {ref }}$ by accounting for the number $n=N_{b} / N_{\text {ref }}$ of approximately statistically-independent segments of size $N_{\text {ref }}$ at which crossslip can initiate. The appeal of weakest-link scaling is that it avoids the need for random walk calculations and thus facilitates the prediction of the distribution of $\Delta E_{\text {act }}$ for different segment lengths based on accessible inputs (e.g. smaller atomistic simulations; analytic models based on solute/dislocation energies, etc.). Weak-link scaling enables the use of such reduced-order models to make on-the-fly predictions for cross-slip probabilities in higher-scale simulations such as Discrete Dislocation Dynamics models.

Let $P\left(\Delta E_{\text {act }}, N_{\text {ref }}, \tau\right)$ be the cumulative distribution of $\Delta E_{\text {act }}$ for length $N_{\text {ref }}$ at the given stress $\tau$. The probability that all of the $n=N_{b} / N_{\text {ref }}$ segments have an activation energy greater than $\Delta E_{\text {act }}$ is $\left(1-P\left(\Delta E_{\text {act }}, N_{\text {ref }}, \tau\right)\right)^{\left(N_{b} / N_{\text {ref }}\right)}$. Therefore, the cumulative probability $P\left(\Delta E_{\text {act }}, N_{b}, \tau\right)$ for length $N_{b}$ is

$$
P\left(\Delta E_{\mathrm{act}}, N_{b}, \tau\right)=1-\left(1-P\left(\Delta E_{\mathrm{act}}, N_{\mathrm{ref}}, \tau\right)\right)^{\left(N_{b} / N_{\mathrm{ref}}\right)},
$$

This analysis follows standard weakest-link analysis, see for example Ref. [47]. To test this hypothesis, we carried out further random walk calculations with lengths $N_{b}=5000$ and $N_{b}=$ 
10000. We then used the random walk data from calculations at $N_{b}=500$ as reference, i.e. $N_{\text {ref }}=500$. Calculations were carried out for $\mathrm{Cu}+33$ at.\% $\mathrm{Ni}, \mathrm{Al}+06$ at. $\% \mathrm{Mg}$ and $\mathrm{Ni}+15$ at.\% $\mathrm{Al}$.

Fig. 11 shows the cumulative distribution functions for the cross-slip activation energy for $5 \mathrm{MPa}$ Schmid stress on the cross-slip plane, and also $50 \mathrm{MPa}$ for $\mathrm{Cu}+33$ at. $\% \mathrm{Ni}$, for $N_{b}=$ 500, $N_{b}=5000$, and $N_{b}=10000$. The figure also shows the predictions for $N_{b}=5000$ and $N_{b}=10000$ as obtained from weak-link scaling. The weak-link scaling is very accurate, within fractions of a percent, in all cases. The agreement is achieved because, even at the lower stress level, all large barriers have been eliminated within the length $N_{b}=500$.

Fig. 12 shows the cumulative distribution functions for the cross-slip activation energy at both $5 \mathrm{MPa}$ Escaig stress, for $\mathrm{Cu}+33$ at.\% $\mathrm{Ni}$ and $\mathrm{Ni}+15$ at.\% Al. As can be expected from Fig. 9 and Fig. 10, the weakest-link prediction is not accurate at this low stress because the largest barriers can be encountered at lengths larger than the reference length $N_{b}=500$. Thus, higher activation energies are underestimated. The error is smaller in $\mathrm{Cu}+33$ at.\% Ni because the characteristic stress calculated with Eq. 17 is only $\tau_{\text {cross }}^{\text {Esc }}=6.6 \mathrm{MPa}$ for $N_{b}=5000$ and $\tau_{\text {cross }}^{\text {Esc }}=$ 4.7 $\mathrm{MPa}$ for $N_{b}=10000$, close to $5 \mathrm{MPa}$. Nevertheless, the differences in median values are only $-0.12 \mathrm{eV}$ and $-0.08 \mathrm{eV}$, respectively, and so the weak-link scaling captures much of the shift of the activation barriers to lower values, but overestimates the shift.

At $50 \mathrm{MPa}$ Escaig stress, the prediction of weak-link scaling is excellent. At this stress level, all large barriers are overcome well within the length of $N_{b}=500$, making the weak-link model applicable. This is consistent with the estimates for the characteristic stresses of $\tau_{\text {cross }}^{\text {Esc }}=20.8 \mathrm{MPa}$ and $14.8 \mathrm{MPa}$ for $N_{b}=5000$ and $N_{b}=10000$, respectively, in $\mathrm{Ni}+15$ at.\% Al.

\subsection{Predictions based on zero stress data}

At sufficiently high stresses, Eq. 18 allows for the prediction of the activation energy distributions of very long dislocations based on knowledge of the distribution at length $N_{\text {ref }}$. However, the reference distribution needs to be calculated at the given applied stress, and this distribution is not readily accessible. Making predictions would be easier if the reference distribution at zero stress could be used, because this distribution is computable from the underlying solute/dislocation and solute/solute interaction energies. At zero stress, however, $N_{\text {ref }}$ must then be small so that the reference distribution does not have a large tail at high energy barriers. This suggests using the distribution at the length scale of cross-slip nucleation, i.e. $N_{b} \approx 40$. This length is also accessible by atomistic simulations if suitable potentials are available.

The effect of stress can then be approximated by shifting the reference distribution to lower energies before scaling. To capture the average stress effect, we shift the distribution by the deterministic change of $\Delta E_{\text {act,avg, }}$ i.e. $\Delta E_{\text {act,avg }}(\tau)-\Delta E_{\text {act,avg, }}$, which can be obtained from Eq. 16. However, not all energy levels should be shifted by the same amount. Low barriers typically correspond to paths where the energy barrier occurs at small cross-slip segment lengths, see Appendix D. These barriers are relatively insensitive to the work done by the stress in extending
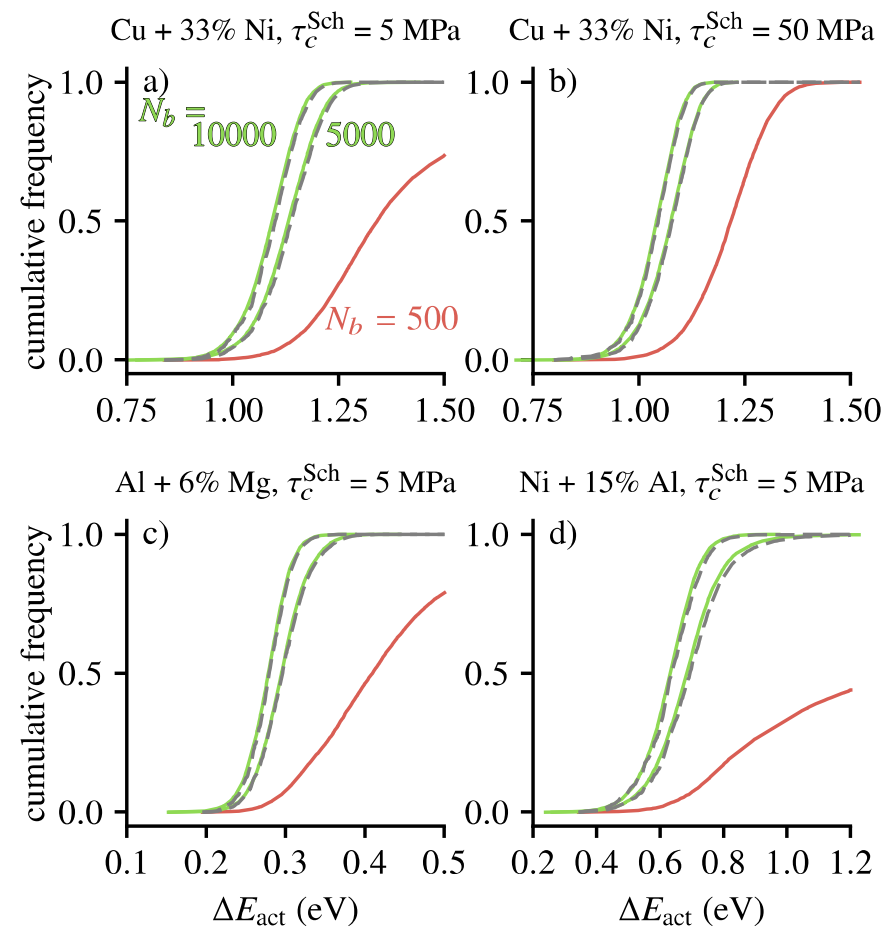

Figure 11: Testing the validity of the weakest-link scaling relation (Eq. 18); lines: cumulative distributions of $\Delta E_{\text {act }}$ at 5 or $50 \mathrm{MPa}$ Schmid stress on the cross-slip plane, in different alloys (a-c) and for different lengths $N_{b}$; red: distribution for $N_{b}=500$; green: distributions for $N_{b}=5000$ and $N_{b}=10000$; red and green data were calculated with the random walk model; dashed lines: predictions using Eq. 18 for $N_{b}=5000$ and $N_{b}=10000$, based on the data for $N_{b}=500$

$\mathrm{Cu}+33 \% \mathrm{Ni}, \tau_{c}^{\mathrm{Esc}}=5 \mathrm{MPa} \quad \mathrm{Cu}+33 \% \mathrm{Ni}, \tau_{c}^{\mathrm{Esc}}=50 \mathrm{MPa}$

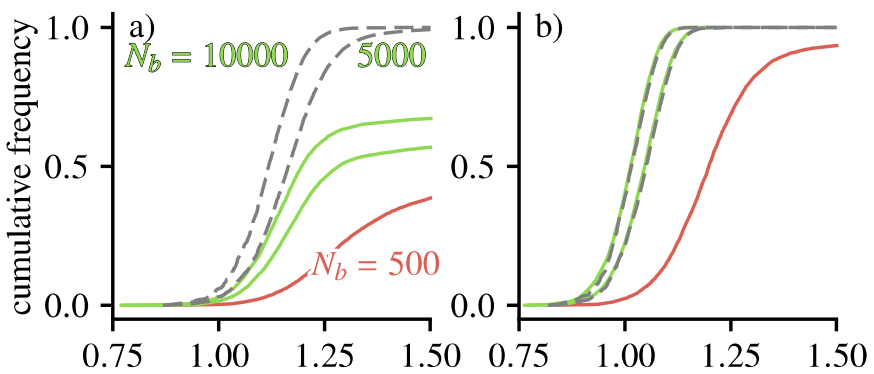

$\mathrm{Ni}+15 \% \mathrm{Al}, \tau_{c}^{\mathrm{Esc}}=5 \mathrm{MPa} \quad \mathrm{Ni}+15 \% \mathrm{Al}, \tau_{c}^{\mathrm{Esc}}=50 \mathrm{MPa}$

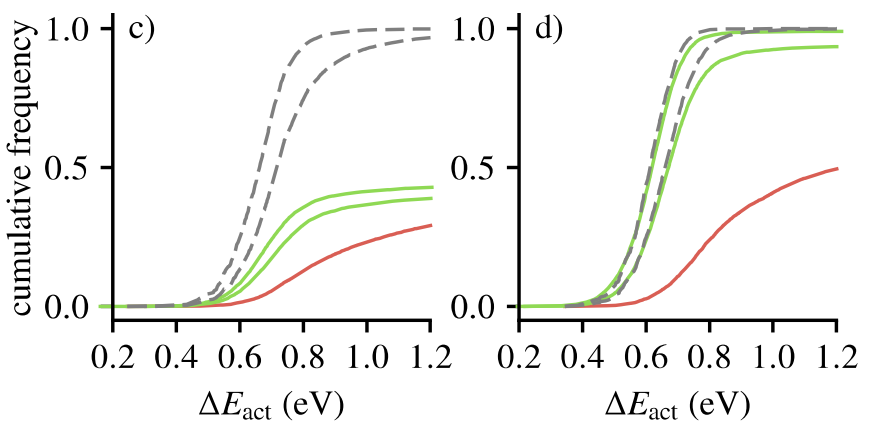

Figure 12: Cumulative distributions of $\Delta E_{\text {act }}$ at 5 or $50 \mathrm{MPa}$ Escaig stress on the cross-slip plane, in different alloys (a-c) and for different lengths $N_{b}$; red: distribution for $N_{b}=500$; green: distributions for $N_{b}=5000$ and $N_{b}=10000$; red and green data were calculated with the random walk model; dashed lines: predictions using Eq. 18 for $N_{b}=5000$ and $N_{b}=10000$, based on the data for $N_{b}=500$ 
or bowing out the dislocation on the cross-slip plane. Conversely, energy barriers equal to $\Delta E_{\text {act,avg }}$ or higher likely correspond to paths where the maximum occurs later, and these see the full stress effect. To approximate this difference in sensitivity, we make the shift a linear function of $\Delta E_{\text {act }}$ for $\Delta E_{\text {act }}<\Delta E_{\text {act,avg, }}$ i.e. $P\left(\Delta E_{\text {act }}, N_{\text {ref }}, \tau\right) \rightarrow P\left(\Delta E_{\text {act }}-\Delta E_{\text {shift }}, N_{\text {ref }}, 0\right)$ with

$$
\begin{aligned}
& \frac{f_{\mathrm{c}}-1}{2} \Delta E_{\mathrm{act}, \mathrm{avg}}+
\end{aligned}
$$

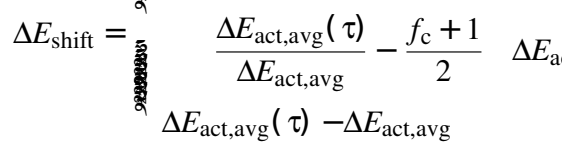

$$
\begin{aligned}
& \Delta E_{\text {act }}<\Delta E_{\text {act,avg }} \\
& \Delta E_{\text {act }} \geq \Delta E_{\text {act,avg }}
\end{aligned}
$$

where $\Delta E_{\text {act,avg }}(\tau)$ is the average-alloy activation energy at stress $\tau$, i.e. the maximum of $E_{\text {det }}$. The first term for $\Delta E_{\text {act }}<\Delta E_{\text {act,avg }}$ is the contribution from the change of energy of the constriction on the glide plane, which applies to all paths, and the second term is the contribution from the work on the cross-slip plane, which has only limited effect for paths with low $\Delta E_{\text {act }}$, as explained above. Note that paths with $\Delta E_{\text {act }} \geq \Delta E_{\text {act,avg }}$ see the full stress effect.

Using the activation energy distribution of $40 b$ long lines as reference data, we predicted the corresponding activation energy distributions at a dislocation length of $1000 b$ under Schmid or Escaig stresses of 50, 200 and $600 \mathrm{MPa}$. Note that $N_{b}=1000$ corresponds to a dislocation density of roughly $10^{13} \mathrm{~m}^{-2}$ in the alloys considered here, typical of the range during deformation of engineering alloys. The highest stress level is not necessarily relevant for real alloys. For example, reported stresses in compression tests with $\mathrm{Al}+3 \mathrm{wt} \% \mathrm{Mg}$ at room temperature are below $300 \mathrm{MPa}$ [48]. Cu-Ni single crystals deform in roomtemperature tensile tests under resolved shear stresses not higher than $140 \mathrm{MPa}$ [49]. Nevertheless, it is interesting to check the validity of our approach in the limit of very high stress.

In the following, we discuss predictions for $\mathrm{Cu}+33$ at. $\% \mathrm{Ni}$, $\mathrm{Al}+06$ at. $\% \mathrm{Mg}$ and $\mathrm{Ni}+15$ at.\% Al. Note that these alloys have increasing ratios of $\sigma\left[\Delta E_{\text {end }}\right] / \Delta E_{\text {act,avg }}$, meaning that random fluctuations become more important relative to the deterministic energy. In Fig. 13, the predictions for $\mathrm{Cu}+33$ at.\% $\mathrm{Ni}$ and $N_{b}=1000 b$ are compared to the results of full random walk calculations and the average-alloy activation energies that one would expect based on $E_{\text {det }}$. The different stress types $\tau_{\text {cross }}^{\text {Esc }}$, $\tau_{\mathrm{cross}}^{\mathrm{Sch}}$ and $\tau_{\text {glide }}^{\text {Esc }}$ are considered separately in subfigures a), b) and c). We see that the predictions (dashed lines) are close to the direct results (solid lines) at all stress levels (colors). In some cases, for example at $\tau_{\text {cross }}^{\mathrm{Esc}}=200 \mathrm{MPa}, \Delta E_{\text {act }}$ is overestimated by roughly $0.1 \mathrm{eV}$. Furthermore, the predicted distributions tend to be slightly broader at $200 \mathrm{MPa}$ and $600 \mathrm{MPa}$. However, these errors are small compared to the error one would make by using only the average-alloy activation energy (shown as the cross symbols in the figures). At the median value, for example, the error of the average alloy is typically four to five times larger than the error of the weakest-link prediction. When predicting cross-slip rates, this error would be further amplified by the exponential weighting of $\Delta E_{\text {act }}$ in Eq. 1.

In $\mathrm{Al}+06$ at. $\% \mathrm{Mg}$, the predictions are satisfactory as well,
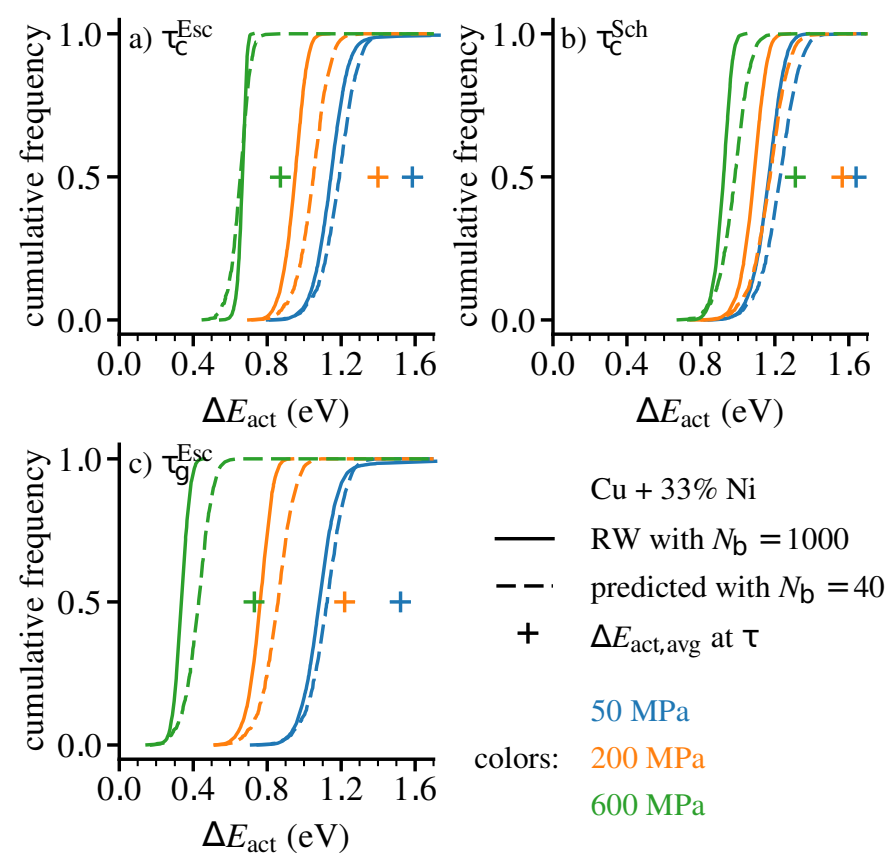

Figure 13: Cumulative distributions of cross-slip activation energies in $\mathrm{Cu}+33$ at. $\% \mathrm{Ni}$ under stress; dashed lines are predictions made with Eq. 18, based on shifted zero-stress distributions for $N_{b}=40$

see Fig. 14. The error is typically less than $0.1 \mathrm{eV}$. At $\tau_{\text {glide }}^{\text {Esc }}=$ $50 \mathrm{MPa}$ and $\tau_{\text {cross }}^{\text {Esc }}=50 \mathrm{MPa}$, the highest $10-15 \%$ of energies are underestimated, because some high barriers have not been eliminated by the stress in the direct calculations. However, the other $90-85 \%$ of energies are predicted accurately, and it are these lower energies which are more important, due to the exponential weighting in Eq. 1.

The mismatch between prediction and direct calculations at $50 \mathrm{MPa}$ Escaig stress is even stronger in $\mathrm{Ni}+15$ at. $\% \mathrm{Al}$, see Fig. 15. $\Delta E_{\text {act }}$ is underestimated above the median value. However, this error is not surprising, since $50 \mathrm{MPa}$ is just above the limit stresses of $\tau_{\text {glide }}^{\text {Esc }}=48 \mathrm{MPa}$ and $\tau_{\text {cross }}^{\text {Esc }}=46 \mathrm{MPa}$ according to Eq. 17. The prediction is nevertheless useful, since it indicates that a significant number of paths have an activation energy of around $0.7 \mathrm{eV}$, hence thermally activated cross-slip is plausible. Based on the average-alloy value of $1.89 \mathrm{eV}$, one would perhaps rule it out. At the other stress levels, the prediction is much more accurate.

\section{Discussion}

We have demonstrated that (i) the standard deviationo $\left[\Delta E_{\text {end }}\right]$ of random energy fluctuations caused by cross-slip in random alloys can be predicted accurately based on solute/dislocation and solute/solute interaction energies; (ii) the random walk model captures the accumulation of energy fluctuations during cross-slip over long lengths, such that (iii) the distribution of the cross-slip activation energies $\Delta E_{\text {act }}$ can be computed for any dislocation length, including the range of practical interest $\left(\sim 10^{3} b\right)$, where direct atomistic calculations are infeasible. We 

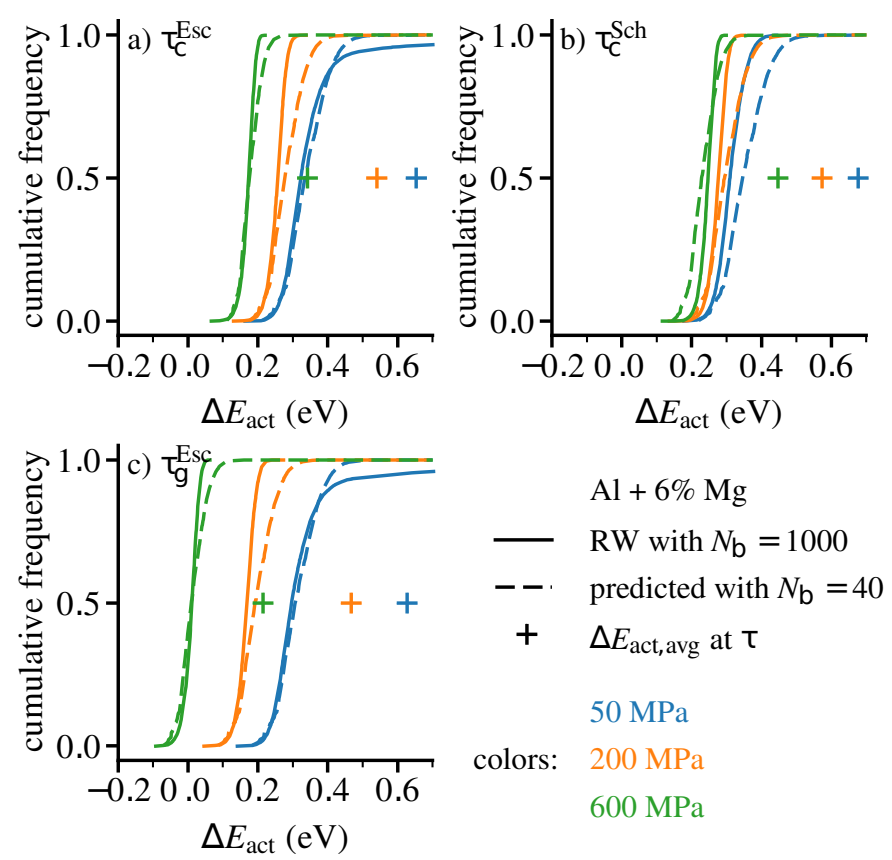

Figure 14: Cumulative distributions of cross-slip activation energies in $\mathrm{Al}+06$ at. $\% \mathrm{Mg}$ under stress; dashed lines are predictions made with Eq. 18, based on shifted zero-stress distributions for $N_{b}=40$
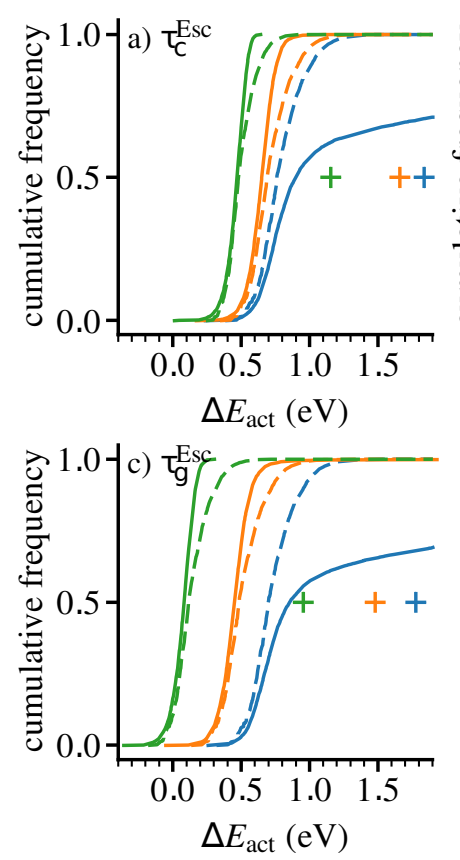

Figure 15: Cumulative distributions of cross-slip activation energies in $\mathrm{Ni}+15$ at.\% Al under stress; dashed lines are predictions made with Eq. 18, based on shifted zero-stress distributions for $N_{b}=40$. The simulated data (solid lines) shows that a $50 \mathrm{MPa}$ Escaig stress is not sufficient to eliminate all high energy barriers. The prediction according to Eq. 18 cannot predict high barriers, hence there is a discrepancy. find that such long dislocations frequently have very high $\Delta E_{\mathrm{act}}$, on the order of several electron volts, but that moderate stresses $\left(10^{0}-10^{1} \mathrm{MPa}\right)$, especially Schmid stresses, eliminate these barriers and lead to activation barriers that are much lower than the average value. We have then shown that cross-slip becomes a weakest-link problem, enabling computation of barriers for long lengths in terms of barriers computed at shorter lengths. We now discuss implications of these results and some further aspects of our model.

First of all, complete cross-slip of long dislocations $\left(10^{3} b\right)$ is unlikely at zero stress, because of the increasing probability of high activation energies. Due to the exponential weighting of $\Delta E_{\text {act }}$ in Eq. 1, these barriers are exceedingly unlikely to be overcome by thermal activation alone. However, when these high barriers are eliminated by an external Escaig or Schmid stress, then $\Delta E_{\text {act }}$ is typically much lower than the value $\Delta E_{\text {act,avg }}(\tau)$ that one would expect based on an average-alloy model. Thus, cross-slip is much faster than expected. For example, in $\mathrm{Cu}+33$ at.\% Ni with a $50 \mathrm{MPa}$ Escaig stress on the glide plane, the median activation energy for a $1000 b$ long dislocation is $1.14 \mathrm{eV}$, whereas the average-alloy estimate is $1.59 \mathrm{eV}$. Assuming constant prefactors in Eq. 1, the rate of cross-slip in the random alloy is roughly 7 orders of magnitude faster than expected based on an averagealloy model. In $\mathrm{Al}+02$ at. $\% \mathrm{Mg}$ and $\mathrm{Ni}+15$ at.\% $\mathrm{Al}$, the rate is increased by 4 and 16 orders of magnitude, respectively.

Under stress, cross-slip becomes a weakest-link problem because the cross-slipped segment expands spontaneously after nucleation. The distribution of $\Delta E_{\text {act }}$ depends on (i) the distribution of $\Delta E_{\text {act }}$ for cross-slip nucleation, which takes place over a length much shorter than the total length, and (ii) how many times this distribution is sampled over the total length. We observed that even small Schmid stresses on the cross-slip plane, on the order of a few MPa, eliminate high barriers and make cross-slip a weakest-link problem. As a consequence, cross-slip of moving dislocations is likely always a weakest-link problem. In order to make the dislocation move, a Schmid stress on the glide plane is required. Except in specific load cases, the applied stress will likely have a Schmid stress component on both the glide and the cross-slip plane. If the applied stress is high enough so that the component on the glide plane causes the dislocation to move, then the component on the cross-slip plane is likely high enough to make cross-slip a weakest-link problem.

In alloys, one additionally has to consider solute strengthening. Recall that we have neglected energy fluctuations during bowing-out, i.e. we have neglected solute strengthening on the cross-slip plane. However, if we assume that the external stress is sufficiently high to make dislocations move on the glide plane, then it is, as before, likely that critical stresses on the cross-slip plane are sufficient, so that a bow-out can be formed.

Internal stresses have to be considered as well. For example, the critical stress for weakest-link scaling can be overcome easily in a pile-up of $n$ dislocations against an obstacle, where the external stress is amplified by a factor equah [50]. Pile-ups were proposed early on as likely sites for cross-slip [51]. Dislocations that do not reside in pile-ups nevertheless experience stresses from other dislocations, e.g. forest dislocations. A particularly interesting scenario is cross-slip of a dislocation in a screw 
dipole, which can lead to annihilation (see Refs. [14, 16, 52] for atomistic simulations of this process). Here, one would expect large attractive stresses.

The easier/faster cross-slip implied by our results here would have several consequences for plastic deformation behavior. Screw dipole annihilation plays a role in fatigue and may determine the saturation stress [53], hence our observations may help to interpret fatigue of alloys. Due to the faster annihilation by cross-slip one should expect larger minimum stable dipole heights as in pure metals, and hence lower saturation stresses. Discrete $[1,54]$ and continuum dislocation dynamics calculations $[2,55,56]$ have demonstrated the importance of cross-slip for dislocation microstructure formation. A cellular microstructure is only observed if cross-slip is activated in such calculations. Thus, one would expect to see a well-developed cellular microstructure in deformed random alloys. Moreover, this microstructure should form more rapidly with strain than in pure metals. Considering the results of Xia and coworkers [56], one would also expect higher yield points and an increased hardening rate. However, it might be difficult to distinguish these effects from other solute strengthening effects.

The case of $\mathrm{Ni}+15$ at. $\%$ shows that low values of $\Delta E_{\text {act }}$ can be realized even in alloys with high $\Delta E_{\text {act,avg }}$ if $\sigma\left[\Delta E_{\text {end }}\right]$ is high and moderate stresses are applied. In High Entropy Alloys [29], where $\sigma\left[\Delta E_{\text {end }}\right]$ can be expected to be high due to compositional disorder, cross-slip should therefore be relatively easy. Indeed, Rao et al. [57] recently observed spontaneous cross-slip in atomistic simulations of regular dislocation slip in a $\mathrm{Co}_{40} \mathrm{Fe}_{16.67} \mathrm{Ni}_{36.67} \mathrm{Ti}_{16.67}$ random alloy at $300 \mathrm{~K}$, even though $\Delta E_{\text {act,avg }}$ is $4.6-4.9 \mathrm{eV}$. The effective value of $\Delta E_{\text {act }}$ was estimated to be $0.3 \mathrm{eV}$. Liu et al. [58] observed that twins in $\mathrm{Al}_{0.1} \mathrm{CoCrFeNi}$ were formed by a mechanism which involves cross-slip of dislocations that were piled up against a stacking fault. These studies show the potential importance of crossslip in HEAs. Somewhat conflicting in this sense is the study of Cao et al. [59], who performed creep tests with a $\mathrm{Al}_{\mathrm{x}} \mathrm{CoCr}$ FeNi ( $x=0.15,0.60)$ HEA and determined activation volumes of $32-52 b^{3}$, which are consistent with a cross-slip mechanism [60], but also high activation energies (3.5-4 eV). Furthermore, Otto and coworkers [61] observed planar slip in CoCrFeMnNi, which seems to be in conflict with our suggestion of easy crossslip. The authors attributed planar slip to short-range order (SRO), which might inhibit cross-slip due to glide plane softening [62]. We have neglected SRO, because we assumed a completely random solute distribution. However, we believe that the present model for random alloys provides a basis for the future investigation of alloys with non-zero SRO. For example, one may speculate that correlations in the solute distributions would reduce $\sigma\left[\Delta E_{\text {act }}\right]$, and hence the frequency and magnitude of favorable energy fluctuations. Furthermore, we note that our model contradicts a model of planar slip by Hong and Laird 20]. They argued that solutes create a friction stress on the partial dislocations, which impedes constriction and hence cross-slip. The friction stress increases with atomic size misfit and solute content, hence it would seem that solute addition should generally impede cross-slip. However, we have shown that one needs to consider solute binding energy fluctuations, which can be positive or negative and thus impede or facilitate cross-slip.

A few other aspects of our model merit discussion. Some approximations were made when modeling the deterministic energy $E_{\mathrm{det}}(i)\left(i=1 . N_{b}\right)$. The current model is appealing because of its simplicity because the energy profile is reduced to two variables, the energy of a single constriction and the cross-slipped length at which the constrictions on the cross-slip plane are approximately fully formed. Both can be determined using atomistic or elastic models. We obtained a satisfactory agreement between $E_{\text {det }}$ and the atomistic data by choosing a formation length of $10 b$. Indeed, the relative energy has almost reached its final value of $\Delta E_{\text {act,avg }}$ at this point, see Fig. 5. Strictly, one would have to choose a value of $20 b$, i.e. half of the total cell length of $40 \mathrm{~b}$, which we identified in our previous publication [13] as the periodic length in $\mathrm{Ni}$ at which $\Delta E_{\text {act,avg }}$ becomes independent of length. However, it is easy to see that the quadratic energy profile would then underestimate the relative energy more strongly in the region of constriction formation. Better approximations of the energy-length profile could be constructed using a different functional form. For example, in Püschl and Schöck's anisotropic-elastic model, the energy of the two half-constrictions on the cross-slip plane varies with cross-slipped length $L$ and dissociation width $d$ approximately as $2 / \pi \arctan (p L / d) 2 E_{c}$, where $2 E_{c}$ is the energy of two wellseparated half-constrictions, and $p$ is a numerical factor in the range $0.8-1.4$. The model of $E_{\mathrm{det}}$ under stress could be improved by including the effect of $\tau_{\text {cross }}^{\text {Esc }}$ on the constriction on the crossslip plane. This could be done by simultaneously scaling the length and the energy of this constriction.

We have also assumed that the ideal Friedel-Escaig mechanism is the operative cross-slip mechanism. This means that the segments on the glide and cross-slip plane are joined by point constrictions. Oren et al. [9] have recently observed a variation of this mechanism in molecular dynamics calculations, where fully constricted segments are formed in the process. Analysis of our atomistic configurations (at zero stress) with the Dislocation Analysis (DXA) algorithm implemented in OVITO [26, 63], shows that such segments occur frequently. In random alloys, lengths of roughly $3 b$ are frequently seen before the transition state. At the transition state, however, the fully constricted length is typically only around $1 b$. Since the length at the transition state is typically short, it is a fair assumption to use the activation energy of ideal Friedel-Escaig cross-slip for $\Delta E_{\text {act,avg }}$.

Three more subtle assumptions were made when modeling the random energy. First, by assigning a random energy change to $1 b$ segments, we associate solute-dislocation binding energies with such short segments. In reality, however, the binding energy quantifies the interaction between the solute and an infinitely long dislocation. The energy has contributions from atoms whose distance to the solute atom along the dislocation line is greater than $1 b$. However, the interaction energy drops of rather quickly, due to the $1 / r^{2}$ scaling of the solute stress field. Moreover, a sufficiently long line is realized after crossslip of a few $1 b$ segments. These are the likely reasons why our approximation does not have a significant effect on the results. Another approximation was made in the derivation of $\sigma\left[\Delta E_{\text {end }}\right]$. The contributions to $\Delta E_{\text {end }}$ from solute-dislocation 
and solute-solute binding are sums of energies times occupation variables, which are Bernoulli random variables. Hence, these contributions are discrete random variables. Here, we assume that they are normally distributed, even for $1 b$ segments. In the case of the contribution from solute-solute binding, this approximation is expected to deteriorate when the number of contributing pairs is low, in a similar way as the approximation of a binomial distribution $B(n, p)$ with a normal distribution deteriorates at low $n$ and $p$. The slight overestimation of $\sigma\left[\Delta E_{\text {act }}\right]$ of $\mathrm{Ni}+02$ at.\% $\mathrm{Al}$ is perhaps an indication of this problem. Furthermore, we have calculated solute-dislocation binding energies using the dislocation core structure at zero stress. Thus, our model of $\sigma\left[\Delta E_{\text {end,s-d }}\right]$ does not account for a change of dissociation width $d$ under stress. Since the highest contributions to $\Delta E_{\text {end,s-d }}$ come from the immediate core region of the partial dislocations, the error of approximation should be small, but one must be careful in alloys where there is significant interaction between solutes and the stacking fault, and at high Escaig stresses. Similarly, $\sigma\left[\Delta E_{\text {end,s-s }}\right]$ was calculated using the zero-stress value of $d$. However, here one could simply use a stress-dependent value.

\section{Conclusion}

In FCC random solid solutions at moderate stresses, the barriers for cross-slip of long dislocation are much lower than expected based on average-alloy properties and models applicable to the elemental metals. Therefore, ruling out cross-slip as an active mechanism on the basis of the average value of the stacking fault energy alone is likely not valid. The analyses here provide a means of assessing the realistic/operative barriers in solid-solution alloys at different levels of approximation. Independent of the level of approximation, the fundamental driver for low activation barriers is due to local fluctuations in solute compositions due to solute/dislocation and solute/solute interactions. There are many implications of these results for interpreting deformation behavior in solid-solution alloys, encompassing both traditional alloys in the dilute-to-moderate concentration regime as well as the rapidly-emerging High Entropy Alloys.

\section{Outlook}

We expect that the results of the current work will be useful for a number of related research areas. For example, our results could help to improve work hardening models that incorporate dislocation density as a state variable. Some models use crossslip as a dislocation sink mechanism and thus require a value for $\Delta E_{\text {act }}$, see Refs. [3, 4, 48, 64]. As we have shown, there is no single value of $\Delta E_{\text {act }}$ in solid solution alloys or HEAs, but a (length- and stress-dependent) distribution. The total rate of cross-slip at a particular stress level can perhaps be calculated by integrating contributions from all relevant segment lengths and energy levels. Weakest-link scaling (Eq. 18 and Eq. 19) could perhaps simplify this problem. Similarly, our results could help to revise cross-slip rules for Discrete Dislocation Dynamics (DDD) models, in order to enable simulation of solid solution alloys and HEAs. In some models, screw dislocation segments of length $L$ cross-slip with a probability $P$, which has the form $L \exp \left(-\Delta E_{\text {act }} / k T\right)[1,65] . L$ in the pre-exponential factor can be seen as a consequence of the fact that, in a pure metal, crossslip could nucleate anywhere along the line. In the case of solid solution alloys, $\Delta E_{\text {act }}$ should be sampled from the distribution for the corresponding length and stress, which could be obtained on the fly from a reference distribution using Eqs. 18 and 19. $L$ in the pre-exponential factor should be removed. The rule for selecting paths that was presented in Sec. 3 implies that there is a unique cross-slip path for each dislocation in its current solute environment, i.e. the nucleation point is not arbitrary anymore.

Another direction for further research is the effect of interstitials and vacancies on cross-slip. In particular, the role of interstitials is not clear. On the one hand, they distort the lattice more strongly than substitutional solutes, hence one would expect a stronger interaction with the cross-slipping dislocation. On the other hand, the concentration of interstitials is typically lower. For example, the maximum concentration of $\mathrm{C}$ in austenitic $\mathrm{Fe}$ is about $2 \%$. The role of these defects can perhaps be studied using a modified version of the random walk model. For a completely random distribution of vacancies, one could calculate $\sigma\left[\Delta E_{\text {end }}\right]$ as described in Sec. 2. In the case of interstitial atoms, one would have to repeat the analysis in Appendix A for interstitial sites in order to derive an equivalent to Eq. 6. However, in both cases, segregation may have to be considered. The present model is then not directly applicable, since the solute distribution was assumed to be completely random.

Furthermore, the random walk approach could be useful for studying solute effects on other mechanisms of dislocation motion, e.g. glide by the kink-pair mechanism or cross-slip in hexagonal close-packed metals. However, some modifications are required. For the calculation of $\sigma\left[\Delta E_{\mathrm{end}, \mathrm{s}-\mathrm{s}, X Y}\right], \zeta / b$ in Eq. 4 needs to be replaced by the correct unit cell length along the dislocation line for the given crystal structure. Similarly, $\sigma\left[\Delta E_{\text {end,s-s, } X Y}\right]$ should be derived for the given crystal structure and stacking fault using the approach in Appendix A. Furthermore, Eq. 16 needs to be modified to describe different origins of $E_{\mathrm{det}}$, e.g. from kink-pair formation.

Finally, there are some problems related to cross-slip which are beyond the current work, but which should be studied to improve current understanding of this important phenomenon. For example, there is currently no analytical model for the preexponential factor $v$ of Eq. 1 in alloys, neither has the value of this factor in alloys been measured directly, for example using direct atomistic calculations as in Refs. [ 9, 14]. Moreover, it would be useful to study anharmonic/entropic contributions to the cross-slip rate. Entropic effects have been observed to be significant in the case of dislocation nucleation [66] and motion of a dislocation through a solute field [67]. Finally, we note that there are still only few experimentally determined values for $\Delta E_{\text {act }}$ of FCC cross-slip.

\section{Acknowledgment}

Support for this work was provided by the European Research Council through the Advanced Grant "Predictive Compu- 
tational Metallurgy”, ERC Grant agreement No. 339081 - PreCoMet. Furthermore, support was provided by EPFL through the use of the facilities of its Scientific IT and Application Support Center.

\section{Appendix A. Extended solute pair model}

Our goal is to calculate the standard deviation of the change in solute-solute binding energies due to formation and destruction of solute pairs in the stacking fault ribbon during cross-slip. In the following, we first briefly recapitulate our previous model [13], which accounts only for first nearest neighbor pairs, and then derive a new model that includes first to eight nearest neighbors.

Previously, the standard deviation was approximated as

$$
\sigma\left[\Delta E_{\mathrm{end}, \mathrm{s}-\mathrm{s}, X Y}\right]=U_{\mathrm{s}-\mathrm{s}, X Y, 1} \sigma\left[\Delta N_{\mathrm{s}-\mathrm{s}}\right],
$$

where $U_{\mathrm{s}-\mathrm{s}, X Y, 1}$ is the binding energy of a first nearest neighbor solute pair, and $\Delta N_{\mathrm{s}-\mathrm{s}}$ is the change in the number of such pairs during cross-slip. Pairs are cut and formed in the stacking fault ribbon of the dissociated dislocation, which is destroyed on the glide plane and regenerated on the cross-slip plane. Hence, the variance of $\Delta N_{\mathrm{s}-\mathrm{s}}$ is approximately two times the variance of the change in the number of pairs during formation or destruction of a single stacking fault ribbon. To derive this number, only the solute occupations of the sites in the upper and lower plane of the fault need to be taken into account. Considering only atoms in a unit cell with the orientation given in Fig. A.16a), and averaging over occupations, one obtains

$$
\sigma\left[\Delta N_{\mathrm{s}-\mathrm{s}}\right]=8 c^{2}(1-c) \frac{\zeta_{d}}{b^{2} \overline{3}},
$$

where $\zeta$ and $d$ are the length and width of the dislocation, respectively, and $c$ is the solute concentration. We emphasize that Eq. A. 2 is only valid if pair cutting/formation in different unit cells is assumed to be independent. If not, correlations between unit cells need to be taken into account. One would then obtain $(1-c)^{2}$ instead of $(1-c)$ in Eq. A.2. Unfortunately, this aspect of the analysis is not discussed in Ref. [13]. In fact, the correlation terms are mistakenly set to zero, which is then equivalent to the assumption that unit cells are independent. In the extended model below, we make this assumption at the outset.

To improve the model, we include higher order neighbor pairs and correlation between pair changes. For this purpose, we write the change $\Delta E_{\text {end,s-s,sf, } X Y}$ in solute-solute binding energy during stacking fault formation in a very general form. Recall that a stacking fault consists of two $\{111\}$ planes with hexagonalclosed packed coordination. In our derivation, we take atoms below the fault, including those in the lower plane of the fault, as fixed. The stacking fault is generated by displacing the atoms above, including those in the upper plane of the fault, by the Burgers vector of a Shockley partial dislocation.

We consider formation/cutting of pairs whose distance (i) is found in the perfect FCC lattice and (ii) is less than the ninth nearest neighbor distance in FCC. The first restriction means that a) unit cell below the stacking fault

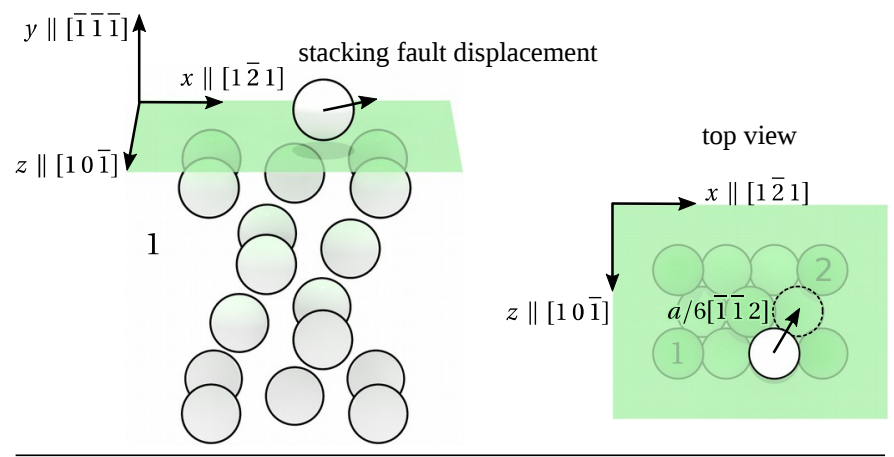

b) neighbors of red atoms

- magenta/cyan/yellow: site lies 1/2/3 layers above the lower plane of the fault - fully colored: pair distance found in perfect FCC lattice (included in analysis)

- black/cross: pair distance not found in perfect FCC lattice (ignored pairs) perfect FCC lattice with stacking fault
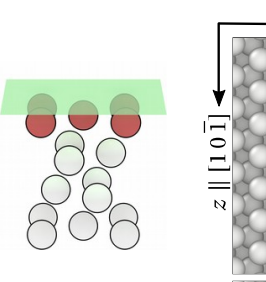
$\rightarrow x \|[1 \overline{2} 1]$
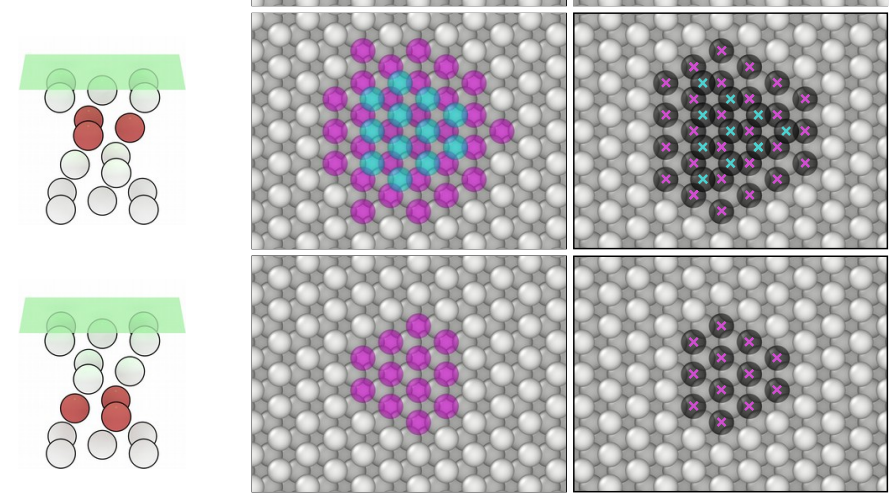

Figure A.16: a) Unit cell used in the derivation of the energy fluctuation due to pair cutting/formation during formation of an intrinsic stacking fault; to form the fault all atoms above the green plane are displaced by $a / 6[\overline{112}]$; b) sites below the stacking fault (red) and their neighbors above (up to eight nearest neighbor distance), before and after generation of the fault; colors distinguish between sites in different $(\overline{111})$-planes; fully colored spheres: sites whose distance to the red atoms is found in the perfect FCC lattice; such pairs are included in the analysis; black with cross: sites whose distance to the red atoms is not found in the perfect FCC lattice; such pairs are neglected

we neglect some pairs which can be identified only in the faulted lattice. The neighbor distances of the pairs that are included in the analysis are listed in Tab. A.2.

Since we restrict the analysis to eighth nearest neighbor distance, we need to consider only a subset of atoms below the fault, namely those in a one unit cell thick layer. Consider a unit cell in the lower region, see Fig. A.16a). The upper plane of the cell corresponds to the lower plane of the fault. The fault can be created by displacing the atoms above by $a / 6[\overline{11} 2]^{5}$. For a stacking fault of length $\zeta$ and width $d$, there are $N_{\zeta} \times N_{d}$ such cells, where $N_{\zeta}=\zeta / b$ and $N_{d}=d / b / \overline{3}$. To get the total change in solute-solute binding energy, we need to sum 
contributions from all atomic sites in these cells. Even lower planes do not need to be considered if we restrict ourselves to eight nearest neighbor pairs. Fig. A.16b) indicates which pairs are included in the analysis. Sites in the unit cell are highlighted red on the left. Neighbors of these sites above the fault are indicated on the right, before and after formation of the fault. Colors distinguish between sites in different $(\overline{111})$ planes. Fully colored spheres indicate sites whose distance to the red atoms is found in the perfect FCC lattice. Black spheres with a cross indicate sites whose distance to the red atoms is not found in the perfect FCC lattice; such pairs are neglected. Note that the ignored pairs all have distances greater than second nearest neighbor distance. For a single unit cell, we count 455 distinct lost and formed pairs which are included in the analysis, and 218 formed pairs which are ignored.

Table A.2: Neighbor distances

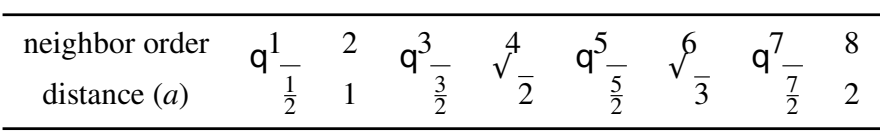

Label the unit cells with indices $i j$, where $i=1 \ldots N_{\zeta}$ and $j=1 . . N_{d}$. Let $\Omega_{\mathrm{lo}, \mathrm{ij}}$ be the set of sites in the unit cell $i j$. There are three or five sites in each $\{111\}$ plane of the unit cell. Some however, lie on a face or in a corner of the cell and are therefore shared with neighboring unit cells. There are only two full sites per plane and eight per unit cell. To generate $\Omega_{\mathrm{lo}, \mathrm{ij}}$, one could either associate eight sites with each cell in a consistent way, or take all sites and introduce extra occupation variables or weighting factors to account for the fact that some sites are associated with multiple unit cells. We choose the latter method, because it allows to take into account more correlations between pair changes within each unit cell. Furthermore, let $\Omega_{\mathrm{hi}}$ be the set of sites above the fault, including the upper plane of the fault. Assign occupation variables $s_{X, i j k}$, where $s_{X, i j k}=1$ if there is a $X$-solute at site $i j k$, and $s_{X, i j k}=0$ otherwise. The $s_{X, i j k}$ are Bernoulli random variables with probability and expectation value equal to the concentration $c_{X}$ of type $X$.

The total change in solute-solute binding energies for pairs formed by $X$-atoms in $\Omega_{\mathrm{lo}, \mathrm{ij}}$ and $Y$-atoms in $\Omega_{\mathrm{hi}}$ is the sum of contributions from all unit cells, i.e.

$$
\Delta E_{\mathrm{end}, \mathrm{s}-\mathrm{s}, \mathrm{sf}, X Y}=\sum_{i}^{N_{\zeta}} \sum_{j}^{N_{d}} \Delta E_{\mathrm{end}, \mathrm{s}-\mathrm{s}, \mathrm{sf}, X Y, i j}
$$

To calculate $\Delta E_{\text {end,s-s, } X Y, i j}$, one sums the changes in solutesolute binding energies for each site in $\Omega_{\mathrm{lo}, \mathrm{ij}}$. Accounting for

\footnotetext{
${ }^{5}$ Slightly different results would be obtained with a displacement $a / 6[\overline{1} 2 \overline{1}]$, i.e. parallel to $x$. The directions $[\overline{11} 2]$ and $[\overline{1} 2 \overline{1}]$ are not equivalent in our model. As we shall see, we consider only neighbor changes for sites in a single unit cell as shown in Fig. A.16a). With this particular choice of sites, the threefold rotation symmetry in the $(\overline{111})$ plane (the plane of the paper) is lost, hence [112] and $[\overline{1} 2 \overline{1}]$ are not equivalent. However, $a / 6[112]$ is the correct displacement corresponding to a Shockley partial of a $1 / 2 a[10 \overline{1}]$ screw dislocation.
}

pairs up to order $N_{\max }$,

$\Delta E_{\mathrm{end}, \mathrm{s}-\mathrm{s}, \mathrm{sf}, X Y, i j}=\sum_{k \in \Omega_{\mathrm{lo}, \mathrm{ij}}} s_{X, i j k} w_{k} \sum_{l}^{N_{\max }} U_{\mathrm{s}-\mathrm{s}, X Y, l} \sum_{m \in \Omega_{\mathrm{hi}}} s_{Y, i j m} \Delta(i, j, k, m, l)$,

where $U_{\mathrm{s}-\mathrm{s}, X Y, l}$ is the binding energy of an $l$-th nearest neighbor $X Y$ pair. The $w_{k}$ are extra occupation variables, which account for the fact that sites on the face or in the corners of the unit cell are shared by multiple unit cells, hence we would have associated only one face or corner site in each plane and unit cell with this cell. They are Bernoulli random variables with probability 1 for sites completely inside the cell, $1 / 2$ for sites on the face of the cell, and $1 / 4$ for corner sites. $\Delta(i, j, k, m, l)$ indicates if a $l$-th order neighbor pair is formed, lost, or preserved, i.e.

$$
\Delta(i, j, k, m, l)=[a(i, j, k, m, l)-b(i, j, k, m, l)],
$$

where $b(i, j, k, m, l)=1$ if the sites are $l$-th nearest neighbors before formation of the fault, and zero otherwise; and $(i, j, k, m, l)=$ 1 if they are $l$-th nearest neighbors after formation of the fault, and zero otherwise.

The variance of $\Delta E_{\text {end,s-s,sf, } X Y}$ is

$$
\operatorname{Var}\left[\Delta E_{\text {end,s-s,sf }, X Y}\right]=\Delta E_{\text {end,s-s,sf, } X Y^{2}}-\Delta E_{\text {end,s-s,sf }, X Y}{ }^{2},
$$

where $h . i$ indicates the average with respect to $s_{X, i j k}$ and $s_{Y, i j k}$. However, a direct calculation would be intractable due to the large number of terms involved. We simplify the problem by making $\Delta(i, j, k, m, l)$ independent of $i$ and $j$. Neighbor changes are analyzed only once, for all atoms in the unit cell shown in Fig. A.16a), i.e.

$$
\Delta(i, j, k, m, l) \equiv \Delta^{c}(k, m, l)=\left[a^{c}(k, m, l)-b^{c}(k, m, l)\right],
$$

where a superscript $c$ is used to refer to the reference cell. Thus, we consider only subsets $\Omega_{\mathrm{lo}}^{c} \subset \Omega_{\mathrm{lo}, \mathrm{ij}}$ and $\Omega_{\mathrm{hi}}^{c} \subset \Omega_{\mathrm{hi}}$. By using $\Delta^{c}(k, m, l)$ instead of $\Delta(i, j, k, m, l)$ we still capture some correlations. For example, we account for exchange of neighbor sites between the sites in the reference unit cell. However, we ignore exchanges between sites in different unit cells with different $i$ and $j$. The numbers $\Delta^{c}(k, m, l)$ were determined by finding the neighbors of sites in a unit cell as shown in Fig. A.16a) before and after insertion of the fault. The crystals were generated with LAMMPS and the distance-based selection could be done conveniently using OvITO [26].

We can now write for the second term in Eq. A.6, noting that $s_{X, i j k}$ and $s_{Y, i j m}$ are independent in a random solid solution,

$$
\begin{aligned}
& \Delta E_{\mathrm{end}, \mathrm{s}-\mathrm{s}, \mathrm{sf}, X Y}{ }^{2}=\sum_{i} \sum_{j}^{N_{\zeta}} \sum_{j} \Delta E_{\mathrm{end}, \mathrm{s}-\mathrm{s}, \mathrm{sf}, X Y, i j}
\end{aligned}
$$

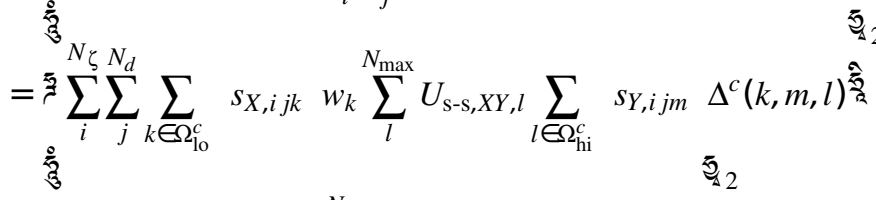

$$
\begin{aligned}
& =\sqrt[5]{\frac{5}{3}} N_{\zeta} N_{d} c_{X} c_{Y} \sum_{k \in \Omega_{\mathrm{lo}}^{c}} w_{k} \sum_{l}^{N_{\max }} U_{\mathrm{s}-\mathrm{s}, X Y, l} \sum_{l \in \Omega_{\mathrm{hi}}^{c}} \Delta^{c}(k, m, l) \text { 旁 }
\end{aligned}
$$


Define

$$
\beta\left(\left\{w_{k}\right\}\right) \equiv \sum_{k \in \Omega_{\mathrm{lo}}^{c}} w_{k} \sum_{l}^{N_{\max }} U_{\mathrm{s}-\mathrm{s}, X Y, l} \sum_{l \in \Omega_{\mathrm{hi}}^{c}} \Delta^{c}(k, m, l),
$$

then

$$
{ }_{\Delta N_{\mathrm{s}-\mathrm{s}, \mathrm{sf}}^{\mathrm{loss}}}^{\mathrm{E}}{ }^{2}=N_{\zeta} N_{d} c_{X} c_{Y} \beta\left(\left\{w_{k}\right\}\right)^{2} .
$$

The first term in Eq. A.6 involves the square of a sum and can be expanded as follows. For the sake of brevity we write $\alpha_{i j} \equiv \Delta E_{\text {end,s-s,sf }, X Y, i j}$. Thus

$$
\begin{aligned}
& \Delta E_{\mathrm{end}, \mathrm{s}-\mathrm{s}, \mathrm{sf}, X Y}^{2}=\sum_{i}^{* 1} \sum_{j}^{N_{\zeta} N_{d}} \alpha^{\#_{2}+}
\end{aligned}
$$

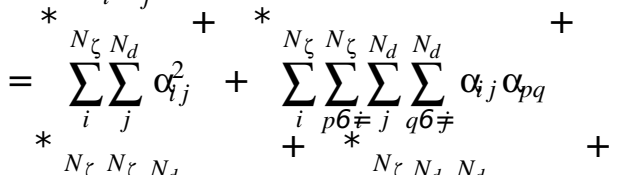

$$
\begin{aligned}
& +\sum_{i} \sum_{p \sigma \dot{\xi}}^{N_{\zeta}} \sum_{\zeta}^{N_{\zeta}} \alpha_{i j} \alpha_{p j}+\sum_{i} \sum_{j} \sum_{q \sigma \dot{\mp}}^{N_{\zeta} N_{d}} \alpha_{i j} \alpha_{i q}
\end{aligned}
$$

The variables $\alpha_{i j}, \alpha_{p q}, \alpha_{p j}$ and $\alpha_{i q}$ are independent, hence

$$
\begin{aligned}
& \Delta E_{\mathrm{end}, \mathrm{s}-\mathrm{s}, \mathrm{sf}, X Y}^{2}=\sum_{i}^{N_{\zeta}} \sum_{j}^{N_{d}} \alpha_{i j}^{2}+\sum_{i}^{N_{\zeta}} \sum_{p \sigma \dot{\xi}}^{N_{\zeta}} \sum_{j}^{N_{d}} \sum_{q \sigma_{\dot{⿰}}}^{N_{d}} \alpha_{i j} \quad \alpha_{p q} \\
& +\sum_{i} \sum_{p \zeta \dot{ }}^{N_{\zeta}} \sum_{j}^{N_{\zeta}} \alpha_{i j} \quad \alpha_{p j}+\sum_{i} \sum_{j}^{N_{\zeta}} \sum_{q \sigma \dot{⿰}}^{N_{d}} \alpha_{i j} \quad \alpha_{i q} .
\end{aligned}
$$

For the last three terms, one obtains

$$
\begin{aligned}
& \sum_{i}^{N_{\zeta}} \sum_{p 6 \dot{\xi}}^{N_{\zeta}} \sum_{j}^{N_{d}} \sum_{q \sigma \dot{\mp}}^{N_{d}} \alpha_{i j} \quad \alpha_{p q}=N_{\zeta} N_{\zeta}-1 \quad N_{d}\left(N_{d}-1\right) \\
& \times\left(c_{X} c_{Y} \beta\left(\left\{w_{k}\right\}\right)\right)^{2}, \\
& \sum_{i}^{N_{\zeta}} \sum_{p \zeta \risingdotseq j}^{N_{\zeta}} \sum_{N_{d}}^{\alpha_{i j}} \quad \alpha_{p j}=N_{\zeta} N_{d} N_{\zeta}-1 \quad\left(c_{X} c_{Y} \beta\left(\left\{w_{k}\right\}\right)\right)^{2} \text {, } \\
& \sum_{i}^{N_{\zeta}} \sum_{j}^{N_{d}} \sum_{q \sigma \dot{ }}^{N_{d}} \alpha_{i j} \quad \alpha_{i q}=N_{\zeta} N_{d}\left(N_{d}-1\right)\left(c_{X} c_{Y} \beta\left(\left\{w_{k}\right\}\right)\right)^{2} .
\end{aligned}
$$

The first term in Eq. A.12 is more cumbersome to resolve, because $\alpha_{i j}^{2}$ is a sum of terms that may contain squares of occupation variables, e.g. $s_{X, i j k}^{2} s_{Y, i j m} s_{Y, i j s}$. Since the occupation variables are Bernoulli random variables, the averages of their squares are the same as the averages of the variables themselves, i.e. $c_{X}$ and $c_{Y}$. Each such product contains at least two independent variables, thus the averaged products have the form $c_{X}{ }^{v} c_{Y}{ }^{w}$ with $v+w \in[2,3,4]$. The correct products of concentrations can be substituted for the products of occupation variables using a computer algebra program, e.g. Mathematica [68].
The variance of $\Delta E_{\text {end,s-s, } X Y}$, the change in solute-solute binding energies during cross-slip, is approximately two times the variance of $\Delta E_{\text {end,s-s,sf, } X Y}$. Combining Eq. A.6, Eq. A.13, Eq. A.12 and Eq. A.10, one obtains the preliminary result

$$
\Delta E_{\text {end, }, \mathrm{s}, X Y}=2 \quad N_{\zeta} N_{d}\left(c_{X} c_{Y} \beta\left(\left\{w_{k}\right\}\right)\right)^{2}+\sum_{i}^{N_{\zeta}} \sum_{j}^{N_{d}} \alpha_{i j}^{2} \text {. }
$$

Both terms on the right hand side still contain the extra occupation variables $w_{k}$. They must be averaged over. Recall that the $w_{k}$ are Bernoulli random variables, with probabilities 1 for center sites, $1 / 4$ for corner sites, and 1/2 for sites on the face of the unit cell. Since they are Bernoulli variables, powers of $w_{k}$ are replaced by the corresponding probabilities. Again, a computer algebra program can be used to make these substitutions in Eq. A.14. The final result for $\Delta E_{\mathrm{end}, \mathrm{s}-\mathrm{s}, X Y}$ is quite long. However, all terms are of the form $A U_{\mathrm{s}-\mathrm{s}, X Y, l} U_{\mathrm{s}-\mathrm{s}, X Y, m} c_{X}{ }^{v} c_{Y}{ }^{w}$, where $A$ is an integer, $v, w=1,2$ and $l, m=1, \ldots N_{\max }$. Thus, it can be written using matrix-vector notation, as in Eq. 6,

$$
\begin{aligned}
& \operatorname{Var}\left[\Delta E_{\mathrm{end}, \mathrm{s}-\mathrm{s}, X Y}\right]=\frac{\zeta d}{b^{2}} c_{X} c_{Y} \frac{1}{\left(1+\delta_{X Y}\right)} \\
& \left.\times \mathbf{U}_{\mathrm{s}-\mathrm{s}, X Y}^{\mid}\left[\mathbf{C}_{1}+\mathbf{C}_{2}\left(c_{X}+c_{Y}\right)-\mathbf{C}_{1}+2 \mathbf{C}_{2}\right) c_{X} c_{Y}\right] \mathbf{U}_{\mathrm{s}-\mathrm{s}, X Y},
\end{aligned}
$$

with coefficient matrices

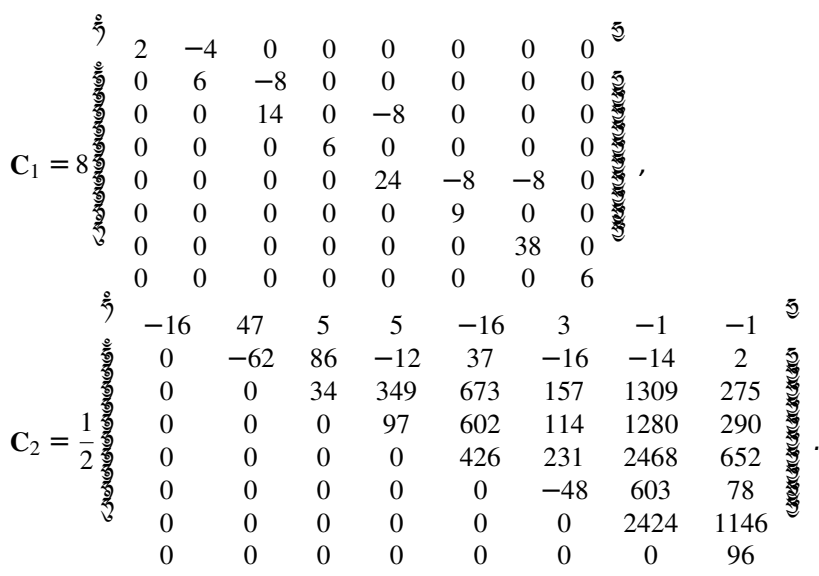

If only first nearest neighbor pairs of like solutes ( $X=Y$, $c=c_{X}=c_{Y}$ ) are considered, one obtains

$$
\operatorname{Var}\left[\Delta N_{\mathrm{s}-\mathrm{s}}\right]=8 c^{2}(1-c) \frac{\zeta_{d}}{b^{2} \overline{3}},
$$

which is equivalent to our previous solution for this special case, Eq. A.2.

\section{Appendix B. Atomistic transition path calculations}

Cross-slip transition paths in average and random alloys were determined using similar methods as in [13]. The L AMMPS [69] package was used for all atomistic calculations. 


\section{Appendix B.1. Configurations for transition path calculations}

The basic geometry was a cylinder with axis parallel to the $z$-direction of the (rectangular) simulation cell. The [1 $\overline{2} 1]$, $[\overline{111}]$ and $[10 \overline{1}]$ crystal directions were parallel to the $x, y$ and $z$-directions of the cell. The cylinder had a shell with a thickness greater than two times the cutoff radius of the potential. Atoms in this region were fixed during simulation runs. The length of the cylinder was $40 b$ and the radius of the inner region $13 \overline{3} a$. The radius was chosen based on the following parameter study. $\Delta E_{\text {act }}$ of cross-slip in average $\mathrm{Ni}+15$ at. $\% \mathrm{Al}$ was calculated for radii in the range $10 \quad \overline{3} a-17.5 \quad \overline{3} a$. Between $12.5 \quad \overline{3} a$ and $17.5 \overline{3} a, \Delta E_{\text {act }}$ increased only by $0.01 \mathrm{eV}$. At the chosen radius of $13 \overline{3} a$, the error in $\Delta E_{\text {act }}$ due to boundary conditions is thus negligible compared to solute effects. A screw dislocation with line direction parallel to [101] and Burgers vector $1 / 2 a[\overline{1} 01]$ was inserted at the center of the cell by imposing the anisotropic-elastic solution for its displacement field by Stroh [70]. During a subsequent energy minimization, the dislocation would dissociate into a pair of Shockley partial dislocations. The Conjugate Gradient (CG) [ 71] and FIRE [ 72] methods were used for energy minimization, see Tab. A.1 in [13] for FIRE parameters. The energy minimization was stopped when the norm of the $3 N$-dimensional force vector fell below $10^{-6} \mathrm{eV} / \AA$. The plane of dissociation was either $(\overline{111})$ (referred to as the glide plane), or ( $\overline{1} 1 \overline{1})$ (cross-slip plane). A dissociated dislocation on the other plane was obtained by making a copy of the pristine cylinder and inserting the screw dislocation at a position that was shifted away from the center along [1 $\overline{2} 1]$ or [ $\overline{121}]$ by fractions of an Ångström. After insertion of the dislocations, the shell atoms of the final state were replaced with the corresponding atoms of the initial state, in order to correct for the small difference in boundary conditions caused by the shifted center.

$252.0 \mathrm{pt}$

\section{Appendix B.2. Transition path calculations with average alloys}

In the case of average alloys, transition path calculations were started with an improved guess for the path, which was constructed as follows. First, a guess for the transition state of the Friedel-Escaig mechanism was generated. A $20 b$ long piece of the initial state and a $20 \mathrm{~b}$ long piece of the final state were combined to form a complete $40 \mathrm{~b}$ long configuration. Smooth constrictions were created at the interface between the two parts by interpolating between the atomic positions of the initial, undissociated, and final state over a length of $30 \AA$ along $z$. The initial guess for the path was created by linearly interpolating between atomic positions in the initial, transition and final state. 32 images were used in the calculation. The transition paths were determined using a custom implementation of the Simplified and Improved String method [73], which is described in Appendix A of Ref. [13]. FIRE was used to move the atoms. The path was re-parameterized by linear interpolation of the atomic coordinates every 100 iterations of the minimizer. The calculation was stopped when the norm of the $3 N$-dimensional vector of displacements between the current re-parameterized state and the previous re-parameterized state was less than $10^{-3} \AA$, or after 600 re-parameterizations (60000 minimization steps).
Appendix B.3. Transition path calculations with random alloys

Random alloy initial and final states were generated as follows. The corresponding average alloy configurations were scaled by the ratio of mean random alloy lattice parameter to average alloy lattice parameter. Thereafter copies were made and converted into true random alloys by randomly replacing the average atom type with real atom types according to the desired concentration. Subsequently, the energy of the configurations was minimized using CG and FIRE. During the minimization, the dislocation could adapt to the random solute environment. Configurations were discarded if the dislocation slipped more than $2 \AA$ away from the center in this process, in order to avoid spurious energy changes associated with image forces. In this way, 100 pairs of initial and final states were created for each composition. Initial guesses for transition path calculations were generated by linear interpolation between the initial and the final state. In contrast to average alloys, no improved initial guess was used, so as to avoid favoring a specific point along the line for cross-slip nucleation.

\section{Appendix B.4. Calculations with Escaig stress}

By resolving the applied stress tensor $\sigma_{i j}(i, j=1,2,3)$ onto the slip planes and partial Burgers vector components, one obtains the following Escaig (superscript Esc) and Schmid (Sch) stresses on the glide and cross-slip planes, respectively:

$$
\begin{aligned}
& \tau_{\text {glide }}^{\text {Esc }}=\sigma_{12}, \\
& \tau_{\text {glide }}^{\text {Sch }}=-\sigma_{23}, \\
& \tau_{\text {cross }}^{\text {Esc }}=\frac{1}{9} \quad 7 \sigma_{12}+2^{\sqrt{ }} \overline{2}\left(\sigma_{11}-\sigma_{22}\right), \\
& \tau_{\text {cross }}^{\text {Sch }}=\frac{1}{9} \quad 2^{\sqrt{ }} \overline{2} \sigma_{13}-\sigma_{23} .
\end{aligned}
$$

Using Hooke's law, one can find a combination of strain components that yields a pure Escaig stress $\tau_{\text {glide }}^{\text {Esc }}$ on the glide plane $\left(\tau_{\text {glide }}^{\text {Sch }}=\tau_{\text {cross }}^{\text {Sch }}=\tau_{\text {cross }}^{\text {Esc }}=0\right)$, for example

$$
\begin{aligned}
\varepsilon_{11} & =-\varepsilon_{22}-\varepsilon_{33}, \\
\varepsilon_{22} & =\frac{7}{8} \overline{\frac{7}{2}} \frac{1}{C_{44}} \tau_{\text {glide' }}^{\text {Esc }} \\
\varepsilon_{33} & =\frac{3}{16} \overline{\frac{3}{2}} \frac{\left(C_{11}-C_{12}-2 C_{44}\right)}{\left(C_{11}-C_{12}\right) C_{44}} \tau_{\mathrm{glide}}^{\text {Esc }} \\
\gamma_{2}=2 \varepsilon_{22} & =\sqrt{\overline{8}} \frac{4}{7} \varepsilon_{22}-\varepsilon_{33}, \\
\gamma_{23} & =\gamma_{3}=0 .
\end{aligned}
$$

For a pure Escaig stress $\tau_{\text {cross }}^{\text {Esc }}$ on the cross-slip plane ( $\tau_{\text {glide }}^{\text {Sch }}=$ $\tau_{\text {cross }}^{\text {Sch }}=\tau_{\text {glide }}^{\text {Esc }}=0$ ), one could impose

$$
\begin{aligned}
\varepsilon_{11} & =-\varepsilon_{22}-\varepsilon_{33}, \\
\varepsilon_{22} & =-\frac{9}{8} \overline{\frac{9}{2}} \frac{1}{C_{44}} \tau_{\text {cross }}^{\text {Esc }} \\
\varepsilon_{33} & =\frac{3}{16} \frac{\left(C_{11}-C_{12}-2 C_{44}\right)}{C_{44}\left(C_{11}-C_{12}\right)} \tau_{\text {cross }}^{\text {Esc }} \\
\gamma_{2}=2 \varepsilon_{2} & =-\overline{8} \varepsilon_{33}, \\
\gamma_{3} & =\gamma_{3}=0 .
\end{aligned}
$$


Using the these equations, Escaig stresses in the range 30 to $300 \mathrm{MPa}$ were imposed on the average alloy initial and final states, as well as the undissociated state. The energy of the initial and the final state was minimized using CG and FIRE. Note that the energy difference between the initial and final state gives $E_{\mathrm{det}}^{\text {Esc }}$ for Eq. 16,

$$
\left.E_{\mathrm{det}}^{\mathrm{Esc}}=(E \text { (final state })-E(\text { initial state })\right) / N_{b},
$$

where $N_{b}=40$ in this case. Transition path calculations were carried out as described above. In the case of the random alloys, calculations were only performed for $150 \mathrm{MPa}$ Escaig stress on the glide or cross-slip plane. The input configurations were generated as described in Appendix B.3, however with the corresponding strained average alloy configurations as templates.

\section{Appendix C. $E_{\text {det }}$ under stress}

Fig. C.17 compares $E_{\text {det }}$ according to Eq. 16 with $N_{c}=10$, and $\tau_{\text {glide }}^{\text {Esc }}=150 \mathrm{MPa}$ or $\tau_{\text {cross }}^{\text {Esc }}=150 \mathrm{MPa}$ to results from atomistic calculations. The cross-slipped length in the atomistic calculations was measured using the dislocation analysis (DXA) algorithm implemented in Ovito [ 26, 63]. The best match is obtained in the case of Ni-15 at.\% Al. In Al-06 at.\% Mg and $\mathrm{Cu}-33$ at. $\% \mathrm{Ni}, E_{\text {det }}$ underestimates the relative energy, especially near the center of the curve. In the case of $\mathrm{Cu}-33$ at. $\% \mathrm{Ni}$, the error is larger when the Escaig stress is applied on the crossslip plane, which is a consequence of neglecting the stress effect on the constriction on this plane. However, the difference between activation energies obtained from the $E_{\text {det }}$ curve and from atomistic calculations is typically less than $0.1 \mathrm{eV}$ for Escaig stresses up to $300 \mathrm{MPa}$, see Fig. C.18.

Our model of the effect of a Schmid stress on the cross-slip plane can be verified by comparing activation energies predicted with Eq. 16 to results from atomistic transition path calculations reported by Kang et al. [8], see Fig. C.19. We also show their predictions based on the Friedel-Escaig model. Note that the material is pure $\mathrm{Ni}$. When evaluating Eq. 16 to find the activation energy, we used the material parameters reported in their publication ( $a=3.52 \AA, C_{11}=244 \mathrm{GPa}, C_{12}=149 \mathrm{GPa}$, $C_{44}=119 \mathrm{GPa}, \Delta E_{\text {act,avg }} \approx 2.27 \mathrm{eV}$ ). The activation energies predicted using the stress-dependent deterministic energy profile are only about $0.06 \mathrm{eV}$ higher than the atomistic energies. Qualitatively and quantitatively, a better match is obtained than with the Friedel-Escaig model. This is not surprising since the zero-stress average alloy activation energy enters as a parameter in our model, whereas the Friedel-Escaig model contains a number of uncertain parameters and is known to not be quantitative $[6,8,74]$.

\section{Appendix D. Correlation between the value of $\Delta E_{\text {act }}$ and the step at which it is realized}

Fig. D.20 shows the frequency with which the maximum energy $\left(\Delta E_{\text {act }}\right)$ was realized on a particular step in random walk calculations with $\mathrm{Cu}+33$ at. $\% \mathrm{Ni}$. Note that $N_{b}=200$, hence there are 200 steps in total. 10000 realizations of the random walk were simulated. $\Delta E_{\text {act }}$ occurs frequently at the beginning or end of the walk. The shape of the distribution is reminiscent of an arcsine distribution, which would be expected for a Wiener process according to Lévy's arcsine law [ 75]. The points in Fig. D.20 indicate the median values of $\Delta E_{\text {act }}$ for those cases where $\Delta E_{\text {act }}$ occured on the corresponding steps. Walks where $\Delta E_{\text {act }}$ occurs early on tend to have a low value of $\Delta E_{\text {act }}$, whereas walks where $\Delta E_{\text {act }}$ occurs near the end tend to have a high value of $\Delta E_{\text {act }}$. Thus, walks with late maxima can be identified as the origin of the tail of high $\Delta E_{\text {act }}$ in the corresponding distribution in Fig. 8. 

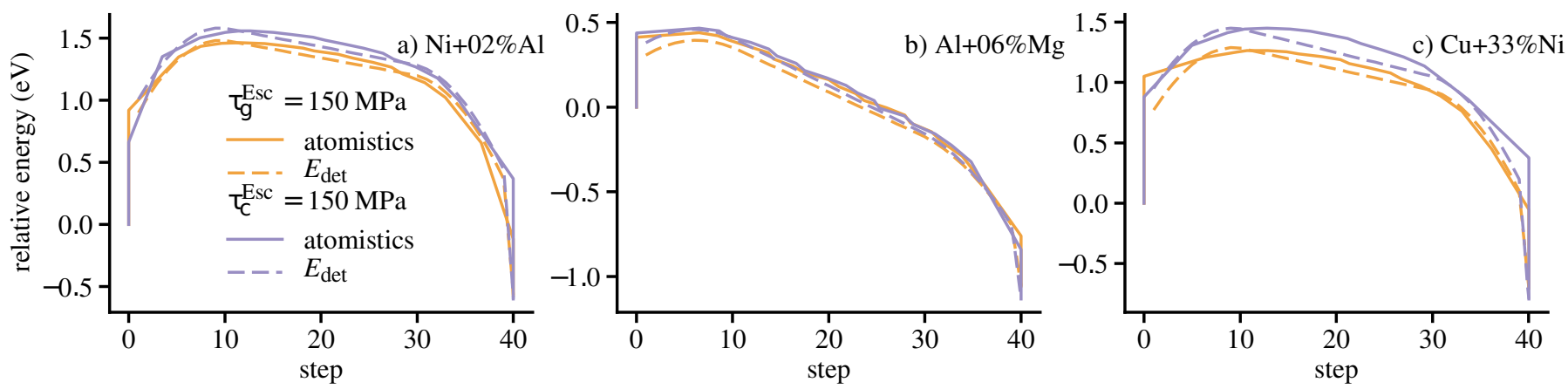

Figure C.17: Relative energy during cross-slip under $150 \mathrm{MPa}$ Escaig stress as a function of step, i.e. length of the cross-slipped segment in Burgers vectors. Solid lines: results from atomistic transition path calculations, see Appendix B.4. Dashed lines: determinisitic energy $E$ det for random walk calculations (Eq. 16).
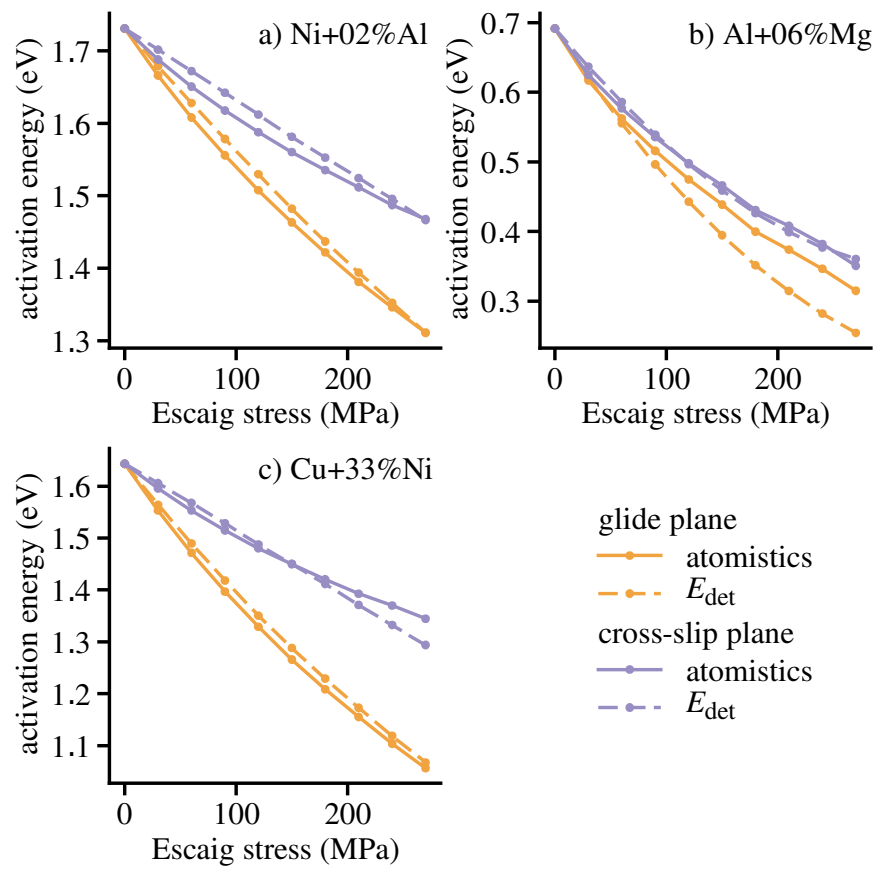

Figure C.18: Cross-slip activation energies as a function of Escaig stress on the glide (Yellow) or cross-slip plane (Violet). Solid lines: results from atomistic transition path calculations, see Appendix B.4. Dashed lines: maximum of the determinisitic energy $E_{\text {det }}$ for random walk calculations (Eq. 16)

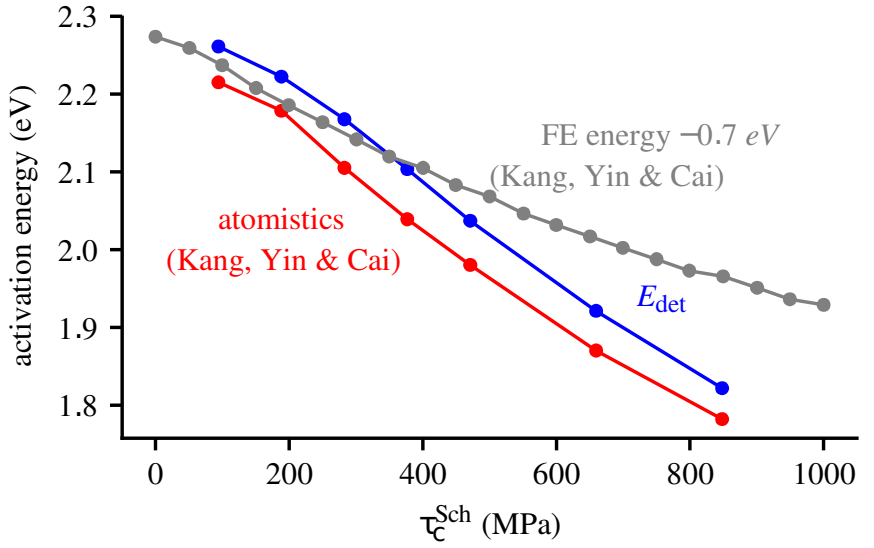

Figure C.19: Cross-slip activation energies in pure $\mathrm{Ni}$ as a function of Schmid stress on the cross-slip plane. Red: atomistic data reported by Kang et al. [8]; gray: values calculated by Kang et al. using their implementation of the FriedelEscaig $[11,12]$ model, shifted by $-0.7 \mathrm{eV}$; blue: maximum of the determinisitic energy $E_{\text {det }}$ for random walk calculations (Eq. 16, evaluated with the material properties reported by Kang et al. ; 


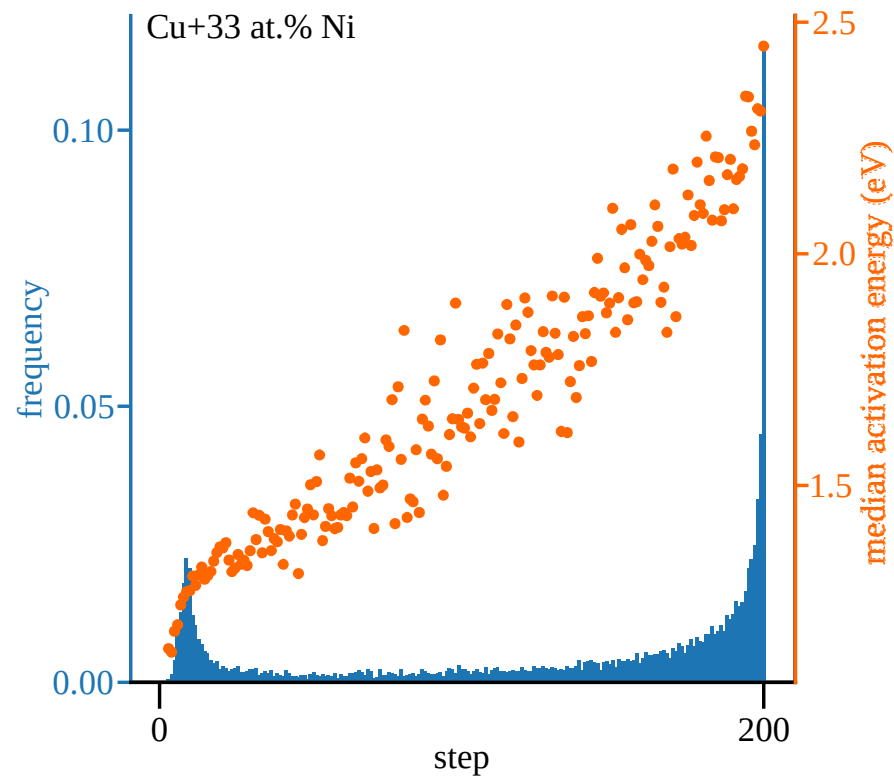

Figure D.20: Blue histogram (left axis): frequency with which the total energy achieves its maximum value $\Delta E_{\text {act }}$ at a particular step in random walk calculations with $\mathrm{Cu}+33$ at. $\% \mathrm{Ni}$ and for $N_{b}=200$ (10000 realizations); orange points (right axis): median values of $\Delta E_{\text {act }}$ for those cases where $\Delta E_{\text {act }}$ occured on the corresponding step

\section{References}

[1] A. M. Hussein, S. I. Rao, M. D. Uchic, D. M. Dimiduk, J. A. El-Awady, Microstructurally based cross-slip mechanisms and their effects on dislocation microstructure evolution in fcc crystals, Acta Materialia 85 (2015) 180-190. doi:10.1016/j.actamat.2014.10.067.

[2] S. Xia, J. Belak, A. El-Azab, The discrete-continuum connection in dislocation dynamics: I. Time coarse graining of cross slip, Modelling and Simulation in Materials Science and Engineering 24 (7) (2016) 075007. doi:10.1088/0965-0393/24/7/075007.

[3] U. F. Kocks, Laws for Work-Hardening and Low-Temperature Creep, Journal of Engineering Materials and Technology 98 (1) (1976) 76-85. doi:10.1115/1.3443340.

[4] W. D. Nix, J. C. Gibeling, D. A. Hughes, Time-dependent deformation of metals, Metallurgical Transactions A 16 (12) (1985) 2215-2226. doi : 10.1007/BF02670420.

[5] K. Karhausen, F. Roters, Development and application of constitutive equations for the multiple-stand hot rolling of Al-alloys, Journal of Materials Processing Technology 123 (2002) 155-166. doi:10.1016/ S0924-0136(02) 00081-X.

[6] W. Püschl, Models for dislocation cross-slip in close-packed crystal structures: A critical review, Progress in materials science 47 (4) (2002) 415-461. doi:10.1016/S0079-6425 (01) 00003-2.

[7] B. R. Raml'irez, N. Ghoniem, G. Po, Ab initio continuum model for the influence of local stress on cross-slip of screw dislocations in fcc metals, Physical Review B 86 (9). doi : 10.1103/PhysRevB.86.094115.

[8] K. Kang, J. Yin, W. Cai, Stress dependence of cross slip energy barrier for face-centered cubic nickel, Journal of the Mechanics and Physics of Solids 62 (2014) 181-193. doi:10.1016/j. jmps . 2013.09.023.

[9] E. Oren, E. Yahel, G. Makov, Kinetics of dislocation cross-slip: A molecular dynamics study, Computational Materials Science 138 (2017) 246-254. doi:10.1016/j.commatsci.2017.06.039.

[10] S. Xu, L. Xiong, Y. Chen, D. L. McDowell, Shear stress- and line lengthdependent screw dislocation cross-slip in FCC Ni, Acta Materialia 122 (2017) 412-419. doi :10.1016/j.actamat.2016.10.005.

[11] J. Friedel, Regarding Seeger's Paper on Work Hardening, in: J. Fisher, W. Johnston, R. Thomson, T. Vreeland, Jr. (Eds.), Dislocations and Mechanical Properties of Crystals, John Wiley \& Sons, Inc., 1957, pp. 330-332.

[12] B. Escaig, Sur le glissement dévié des dislocations dans la structure cu- bique à faces centrées, Journal de Physique 29 (2-3) (1968) 225-239. doi:10.1051/jphys:01968002902-3022500.

[13] W. G. Nöhring, W. A. Curtin, Dislocation cross-slip in FCC solid solution alloys, Acta Materialia 128 (2017) 135-148. doi:10.1016/j. actamat. 2017.02 .027$.

[14] T. Vegge, T. Rasmussen, T. Leffers, O. B. Pedersen, K. W. Jacobsen, Determination of the of rate cross slip of screw dislocations, Physical review letters 85 (18) (2000) 3866. doi:10.1103/PhysRevLett. 85.3866.

[15] T. Vegge, T. Rasmussen, T. Leffers, O. Pedersen, K. Jacobsen, Atomistic simulations of cross-slip of jogged screw dislocations in copper, Philosophical Magazine Letters 81 (3) (2001) 137-144. doi:10.1080/ 09500830010019040 .

[16] S. Rao, D. Dimiduk, J. El-Awady, T. Parthasarathy, M. Uchic, C. Woodward, Screw dislocation cross slip at cross-slip plane jogs and screw dipole annihilation in $\mathrm{FCC} \mathrm{Cu}$ and $\mathrm{Ni}$ investigated via atomistic simulations, Acta Materialia 101 (2015) 10-15. doi : 10.1016/j.actamat.2015.08. 070 .

[17] S. Rao, D. Dimiduk, J. El-Awady, T. Parthasarathy, M. Uchic, C. Woodward, Atomistic simulations of cross-slip nucleation at screw dislocation intersections in face-centered cubic nickel, Philosophical Magazine 89 (3436) (2009) 3351-3369. doi : 10 .1080/14786430903286201.

[18] S. Rao, D. Dimiduk, J. El-Awady, T. Parthasarathy, M. Uchic, C. Woodward, Activated states for cross-slip at screw dislocation intersections in face-centered cubic nickel and copper via atomistic simulation, Acta Materialia 58 (17) (2010) 5547-5557. doi :10.1016/j.actamat. 2010. 06.005 .

[19] S. Rao, D. Dimiduk, J. El-Awady, T. Parthasarathy, M. Uchic, C. Woodward, Spontaneous athermal cross-slip nucleation at screw dislocation intersections in FCC metals and $\mathrm{L}_{2}{ }_{2}$ intermetallics investigated via atomistic simulations, Philosophical Magazine 93 (22) (2013) 3012-3028. doi:10.1080/14786435.2013.799788.

[20] S. I. Hong, C. Laird, Mechanisms of Slip Mode Modification in F.C.C. Solid Solutions, Acta metallurgica et materialia 38 (8) (1990) 1581-1594. doi:10.1016/j.msea.2012.12.044.

[21] S. D. Andrews, H. Sehitoglu, I. Karaman, Constriction energy in the presence of a solute field, Journal of Applied Physics 87 (5) (2000) 2194. doi:10.1063/1.372161.

[22] J.-P. Du, C.-Y. Wang, T. Yu, Cross-slip process in model Ni(Al) solid solution: An embedded-atom method study, Computational Materials Science 91 (2014) 192-199. doi:10.1016/j.commatsci.2014. 04.063 .

[23] M. Wen, S. Fukuyama, K. Yokogawa, Hydrogen-affected cross-slip process in fcc nickel, Physical Review B 69 (17). doi:10.1103/ PhysRevB.69.174108.

[24] M. Wen, S. Fukuyama, K. Yokogawa, Cross-slip process in fcc nickel with hydrogen in a stacking fault: An atomistic study using the embedded-atom method, Physical Review B 75 (14). doi:10.1103/PhysRevB. 75. 144110.

[25] D. Hull, D. Bacon, Introduction to Dislocations, 4th Edition, Butterworth Heinemann, Oxford, 2001

[26] A. Stukowski, Visualization and analysis of atomistic simulation data with OVITO $\{-\}$ the Open Visualization Tool, Modelling and Simulation in Materials Science and Engineering 18 (1) (2010) 015012. doi: 10 . 1088/0965-0393/18/1/015012.

[27] R. W. Smith, G. S. Was, Application of molecular dynamics to the study of hydrogen embrittlement in Ni-Cr-Fe alloys, Physical Review B 40 (15) (1989) 10332-10336. doi:10.1103/PhysRevB. 40.10322.

[28] C. Varvenne, A. Luque, W. G. Nöhring, W. A. Curtin, Average-atom interatomic potential for random alloys, Physical Review B 93 (10) (2016) 104201. doi:10.1103/PhysRevB.93.104201.

[29] J.-W. Yeh, S.-K. Chen, S.-J. Lin, J.-Y. Gan, T.-S. Chin, T.-T. Shun, C.-H. Tsau, S.-Y. Chang, Nanostructured High-Entropy Alloys with Multiple Principal Elements: Novel Alloy Design Concepts and Outcomes, Advanced Engineering Materials 6 (5) (2004) 299-303. doi : 10.1002/adem. 200300567.

[30] C. Varvenne, A. Luque, W. A. Curtin, Theory of strengthening in fcc high entropy alloys, Acta Materialia 118 (2016) 164-176. doi:10.1016/j. actamat.2016.07.040.

[31] G. P. M. Leyson, W. A. Curtin, L. G. Hector, C. F. Woodward, Quantitative prediction of solute strengthening in aluminium alloys, Nature Materials 
9 (9) (2010) 750-755. doi : 10.1038/nmat2813.

[32] G. Leyson, L. Hector, W. Curtin, Solute strengthening from first principles and application to aluminum alloys, Acta Materialia 60 (9) (2012) 3873-3884. doi:10.1016/j.actamat.2012.03.037.

[33] M. S. Daw, M. I. Baskes, Semiempirical, Quantum Mechanical Calculation of Hydrogen Embrittlement in Metals, Physical Review Letters 50 (1983) 1285-1288.

[34] G. Purja Pun, Y. Mishin, Development of an interatomic potential for the Ni-Al system, Philosophical Magazine 89 (34-36) (2009) 3245-3267. doi : 10.1080/14786430903258184.

[35] X.-Y. Liu, J. B. Adams, F. Ercolessi, J. A. Moriarty, EAM potential for magnesium from quantum mechanical forces, Modelling and Simulation in Materials Science and Engineering 4 (1996) 293-303. doi : $10.1088 /$ 0965-0393/4/3/004.

[36] B. Onat, S. Durukanoğlu, An optimized interatomic potential for $\mathrm{Cu}-\mathrm{Ni}$ alloys with the embedded-atom method, Journal of Physics: Condensed Matter 26 (3) (2014) 035404. doi:10.1088/0953-8984/26/3/ 035404

[37] M. Spiegel, L. Stephens, Theory and Problems of Statistics, 4th Edition, McGraw-Hill, New York, 2008.

[38] J. A. Yasi, L. G. Hector, D. R. Trinkle, Prediction of thermal cross-slip stress in magnesium alloys from direct first-principles data, Acta Materialia 59 (14) (2011) 5652-5660. doi : 10.1016/j . actamat. 2011.05 040.

[39] W. Püschl, G. Schoeck, Calculation of cross-slip parameters in fcc crystals, Materials Science and Engineering: A 164 (1) (1993) 286-289. doi : 10.1016/0921-5093 (93) 90679-9.

[40] D. Caillard, J. Martin, Thermally Activated Mechanisms in Crystal Plasticity, 1st Edition, Vol. 8 of Pergamon Materials Series, Pergamon, Amsterdam, 2003.

[41] P. M. Anderson, J. P. Hirth, J. Lothe, Theory of Dislocations, 4th Edition, Cambridge University Press, New York, 2017.

[42] Z. Wu, W. A. Curtin, Mechanism and energetics of $\mathrm{c}+$ a dislocation cross-slip in hcp metals, Proceedings of the National Academy of Sciences 113 (40) (2016) 11137-11142. doi : 10.1073/pnas. 1603966113.

[43] R. O. Scattergood, D. J. Bacon, The Orowan mechanism in anisotropic crystals, Philosophical Magazine 31 (1) (1975) 179-198. doi:10 $1080 / 14786437508229295$.

[44] A. N. Kolmogorov, Sulla determinazione empirica di una legge di distribuzione, Giornale dell'Istituto Italiano degli Attuari 4 (1933) 83-91.

[45] N. V. Smirnov, On the Estimation of the Discrepancy Between Empirical Curves of Distribution for Two Independent Samples, Bulletin mathématiques de l'Université de Moscou 2 (1939) 3-14.

[46] R. A. Fisher, The design of experiments, Oliver and Boyd, Edinburgh, 1935.

[47] D. Krajcinovic, damage mechanics, 1st Edition, Vol. 41 of North-Holland Series in Applied Mathematics and Mechanics, Elsevier, Amsterdam, 1996.

[48] Z. S. Liu, V. Mohles, Solution Strengthening of Various Elements in Aluminium Alloys, Materials Science Forum 794-796 (2014) 473-478. doi:10.4028/www. scientific.net/MSF. 794-796.473.

[49] E. Osswald, Zugversuche an Kupfer-Nickelkristallen, Zeitschrift für Physik 83 (1) (1933) 55-78. doi : 10.1007/BF01331092.

[50] A. H. Cottrell, Theory of dislocations, Progress in Metal Physics 1.

[51] A. Seeger, The Mechanism of Glide and Work Hardening in Face-Centered Cubic and Hexagonal Close-Packed Metals, in: J. Fisher, W. Johnston, R. Thomson, T. Vreeland, Jr. (Eds.), Dislocations and Mechanical Properties of Crystals, John Wiley \& Sons, Inc., 1957, pp. 243-329.

[52] T. Rasmussen, T. Vegge, T. Leffers, O. B. Pedersen, K. W. Jacobsen, Simulation of structure and annihilation of screw dislocation dipoles, Philosophical Magazine A 80 (5) (2000) 1273-1290. doi : 10. 1080/ 01418610008212115

[53] L. M. Brown, Toward a sound understanding of dislocation plasticity, Metallurgical Transactions A 22 (8) (1991) 1693-1708. doi : 10. 1007/ BF02646493.

[54] R. Madec, B. Devincre, L. P. Kubin, Simulation of dislocation patterns in multislip, Scripta materialia 47 (10) (2002) 689-695. doi : 10.1016/ S1359-6462(02) 00185-9.

[55] S. X. Xia, A. El-Azab, A preliminary investigation of dislocation cell structure formation in metals using continuum dislocation dynamics, IOP
Conference Series: Materials Science and Engineering 89 (2015) 012053. doi : 10.1088/1757-899x/89/1/012053.

[56] S. Xia, A. El-Azab, Computational modelling of mesoscale dislocation patterning and plastic deformation of single crystals, Modelling and Simulation in Materials Science and Engineering 23 (5) (2015) 055009. doi:10.1088/0965-0393/23/5/055009.

[57] S. Rao, C. Woodward, T. Parthasarathy, O. Senkov, Atomistic simulations of dislocation behavior in a model FCC multicomponent concentrated solid solution alloy, Acta Materialia 134 (2017) 188-194. doi : 10.1016/j. actamat. 2017.05 .071$.

[58] J. Liu, C. Chen, Y. Xu, S. Wu, G. Wang, H. Wang, Y. Fang, L. Meng, Deformation twinning behaviors of the low stacking fault energy highentropy alloy: An in-situ TEM study, Scripta Materialia 137 (2017) 9-12. doi:10.1016/j.scriptamat.2017.05.001.

[59] T. Cao, J. Shang, J. Zhao, C. Cheng, R. Wang, H. Wang, The influence of $\mathrm{Al}$ elements on the structure and the creep behavior of AlxCoCrFeNi high entropy alloys, Materials Letters 164 (2016) 344-347. doi:10.1016/ j.mat let .2015.11.016.

[60] S. I. Rao, T. A. Parthasarathy, C. Woodward, Atomistic simulation of cross-slip processes in model fcc structures, Philosophical Magazine A 79 (5) (1999) 1167-1192. doi : 10.1080/01418619908210354.

[61] F. Otto, A. Dlouhý, C. Somsen, H. Bei, G. Eggeler, E. George, The influences of temperature and microstructure on the tensile properties of a CoCrFeMnNi high-entropy alloy, Acta Materialia 61 (15) (2013) 5743-5755. doi:10.1016/j.actamat.2013.06.018.

[62] V. Gerold, H. P. Karnthaler, On the origin of planar slip in F.C.C. alloys, Acta Metallurgica 37 (8) (1989) 2177-2183. doi:10.1016/ 0001-6160(89) 90143-0

[63] A. Stukowski, V. V. Bulatov, A. Arsenlis, Automated identification and indexing of dislocations in crystal interfaces, Modelling and Simulation in Materials Science and Engineering 20 (8) (2012) 085007. doi : 10. 1088/0965-0393/20/8/085007.

[64] Y. Estrin, L. S. Tóth, A. Molinari, Y. Bréchet, A Dislocation-Based Model for All Hardening Stages in Large Strain Deformation, Acta Materialia 46 (15) (1998) 5509-5522. doi : 10.1016/S1359-6454(98) 00196-7.

[65] L. P. Kubin, G. Canova, M. Condat, B. Devincre, V. Pontikis, Y. Bréchet, Dislocation Microstructures and Plastic Flow: A 3D Simulation, Solid State Phenomena 23-24 (1992) 455-472. doi : 10. 4028/www. scientific.net/SSP.23-24.455.

[66] S. Ryu, K. Kang, W. Cai, Entropic effect on the rate of dislocation nucleation, Proceedings of the National Academy of Sciences 108 (13) (2011) 5174-5178.

[67] S. Saroukhani, D. Warner, Investigating dislocation motion through a field of solutes with atomistic simulations and reaction rate theory, Acta Materialia 128 (2017) 77-86. doi : 10.1016/j.actamat.2017.02 001.

[68] Wolfram Research, Inc., Mathematica (2016).

[69] S. Plimpton, Fast Parallel Algorithms for Short-Range Molecular Dynamics, Journal of Computational Physics 117 (1995) 1-19. doi: 10.1006/jcph.1995.1039.

[70] A. Stroh, Dislocations and Cracks in Anisotropic Elasticity, Philosophical Magazine 3 (30) (1958) 625-646. doi:10.1080/ 14786435808565804

[71] M. R. Hestenes, E. Stiefel, Methods of Conjugate Gradients for Solving Linear Systems, Journal of Research of the National Bureau of Standards 49 (6) (1952) 409-436. doi : 10.6028/jres.049.044.

[72] E. Bitzek, P. Koskinen, F. Gähler, M. Moseler, P. Gumbsch, Structural Relaxation Made Simple, Physical Review Letters 97 (17). doi : 10. 1103/PhysRevLett. 97.170201.

[73] W. E, W. Ren, E. Vanden-Eijnden, Simplified and improved string method for computing the minimum energy paths in barrier-crossing events, The Journal of Chemical Physics 126 (16) (2007) 164103. doi : 10. 1063/ 1. 2720838

[74] G. Saada, Cross-slip and work hardening of FCC crystals, Materials Science and Engineering: A 137 (1991) 177-183. doi:10.1016/ 0921-5093 (91) 90333-I.

[75] P. Lévy, Sur certains processus stochastiques homogènes, Compositio Mathematica 7 (1940) 283-339. 